\title{
Reflections on the four facets of symmetry: how physics exemplifies rational thinking
}

\author{
Amaury Mouchet \\ Laboratoire de Mathématiques et de Physique Théorique, \\ Université François Rabelais de Tours — CNRS (UMR 7350), \\ Fédération Denis Poisson, \\ Parc de Grandmont 37200 Tours, France \\ mouchet@phys.univ-tours.fr
}

September 17, 2018

\begin{abstract}
In contemporary theoretical physics, the powerful notion of symmetry stands for a web of intricate meanings among which I identify four clusters associated with the notion of transformation, comprehension, invariance and projection. While their interrelations are examined closely these four facets of symmetry are scrutinised one after the other in great detail. This decomposition allows us to carefully examine the multiple different roles symmetry plays in many places in physics. Furthermore, some connections with other disciplines like neurobiology, epistemology, cognitive sciences and, not least, philosophy are proposed in an attempt to show that symmetry can be an organising principle also in these fields.
\end{abstract}

PACS: 11.30.-j, 11.30.Qc, 01.70.+w

Keywords: Symmetry, transformation group, equivalence, classification, invariance, symmetry breaking, reproducibility, objectivity, realism, underdetermination, inference, complexity, emergence, science laws, representations

A shorter version of this manuscript (Mouchet, 2013c) was published in European Physical Journal H. ${ }^{1}$ The parts in blue are additional material to this publication.

\section{Contents}

2 Symmetries, classifications and hierarchies 5

2.1 The four facets of a symmetry . . . . . . . . . . . . . . . . . . . . . 5

2.2 Symmetry as a classification tool and vice versa . . . . . . . . . . . . . . . . . . 6

2.3 Hierarchies . . . . . . . . . . . . . . . . . . . . . . . . . . 7

${ }^{1}$ http://dx.doi.org/10.1140/epjh/e2013-40018-4 
3 Transformations $\quad 7$

3.1 Active and passive physical interpretations of a transformation $\ldots \ldots \ldots \ldots$

3.2 Moving the boundaries . . . . . . . . . . . . . . . . . . . . . . 8

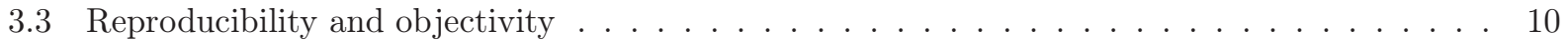

3.4 Relevant groups of transformations . . . . . . . . . . . . . . . . . . . . 11

3.4 .1 Algebraic representations . . . . . . . . . . . . . . . . . . . 11

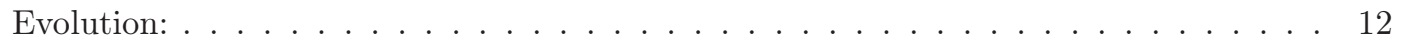

Transformations mixing space and time: . . . . . . . . . . . . . . . . . 12

Exchange of particles: . . . . . . . . . . . . . . . . . . . . . . 12

Gauge transformations: . . . . . . . . . . . . . . . . . . . . . 12

3.4.2 Operational differences between active and passive transformations . . . . . . . . . . . 12

3.5 Perception of space-time transformations: following Henri Poincaré's intuitions . . . . . . . . 13

4 Comprehension and classification 16

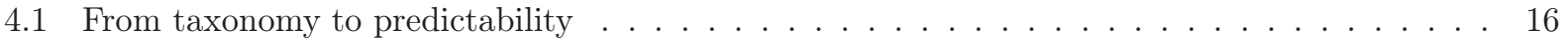

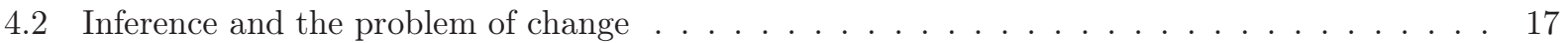

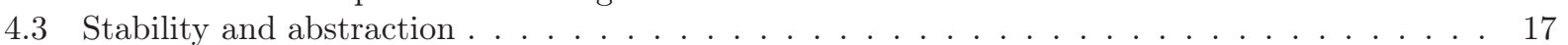

4.4 Different levels of Darwinian selection _ . . . . . . . . . . . . . . . . . . . . . . 19

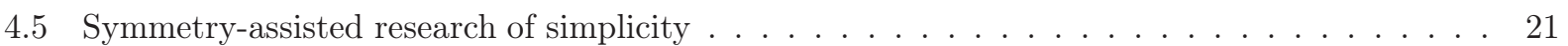

4.5.1 Simplicity as a general evaluation against underdetermination . . . . . . . . . . . . 21

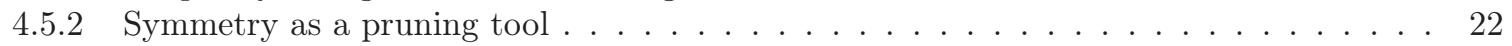

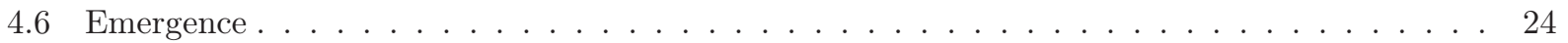

5 Invariance $\quad 25$

5.1 Science laws as quantitative manifestations of invariance . . . . . . . . . . . . . . 26

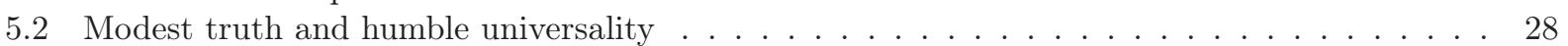

5.3 Epistemic invariance . . . . . . . . . . . . . . . . . . . . . . . . . 29

6 Projection 31

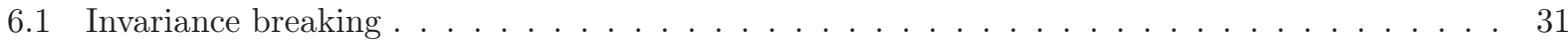

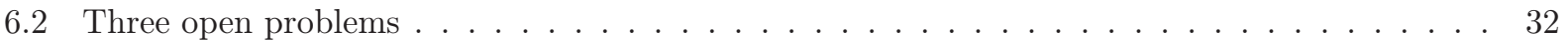

7 Conclusion $\quad 33$ 


\section{Introduction}

During the twentieth century, the development of quantum physics and relativity came with a semantic shift of the word "symmetry" in accordance with the increasing power of such a conceptual instrument. Mathematicians and theoretical physicists have enlarged its meaning far beyond the one it had in the previous centuries (Hon and Goldstein, 2008). Among the multiple uses of symmetry in theoretical physics and more generally in science, I propose to discern four clusters associated with the notion of transformation, comprehension, invariance and projection. After having defined these four facets in the next section, I will discuss them individually with more details in sections $3,4,5$ and 6 respectively. These four notions are more or less explicit and fully recognised in the natural philosophy since its very beginning but, as far as I know, addressed with very loose relations between them, if any. The contemporary theoretical physics point of view on symmetry offers a new and very precise way to tightly bind these notions together and cross-fertilise them. To make a long story short, originally being an aesthetic criterion, symmetry evolves in science into an efficient method of classification before it becomes a powerful theoretical tool to guide and even dictate our way of constructing models and theories. More precisely, symmetry allows

$1^{\circ}$. to build (linear) algebraic representations ${ }^{2}$;

$2^{\circ}$. to constrain yet to unify interactions in the models of theoretical physics;

$3^{\circ}$. to extract universal properties in statistical physics and in non-linear dynamics;

2I use the epithet "algebraic" to reinforce the distinction between a) the precise mathematical meaning of "representation" in the present context (see also $\S 3.4 .1$, Fig. 2 below), b) the "internal" meaning it takes in cognitive science (mental representation) and c) the usual "external" meaning (model, symbol, image, spoken word, written sign, play, etc.). All the three significations share the common point of being associated to a correspondence and can refer to the target, the domain of a mapping, one of their elements or the mapping itself. Some confusion usually raises when the domain and the target are not clearly distinguished. $4^{\circ}$. to solve dynamical equations by reducing the numbers of degrees of freedom and establishing the bridge between integrable and chaotic dynamics;

$5^{\circ}$. to predict selection rules.

Examples of $2^{\circ}$ are the unification of inertial and gravitational forces in the theory of general relativity and the mixing between electromagnetic and weak interactions in the Standard Model of elementary particle physics. Conservation laws are particularly manifest in $4^{\circ}$ and $5^{\circ}$. Above all, the selection rules concern quantum processes but, as constraints on the evolution of a system, they can be found in classical dynamics as well: the conservation of the angular momentum of a particle evolving freely within a circular billiard (with elastic reflexions on its boundaries) explains the impossibility of the inversion of the sense of its rotation around the centre. But any small generic deformation of the billiard boundary, even a small bump, can revert angular momentum (still conserving the energy) and, actually, this breaking of rotational invariance gives room to chaotic motion. In this essay I will have to say more about $1^{\circ}$ in $\S 3.4 .1$ and I will focus on $3^{\circ}$ in $\S 4.5 .2$.

The roles $1^{\circ}-5^{\circ}$ were discovered/invented by physicists essentially during the twentieth century (for a deep insight of these issues see the synthesis by Brading and Castellani, 2003, in particular § 1). These powerful functions of symmetry, far from erasing it, strengthen what is certainly the most important one: the fact that symmetry allows

$6^{\circ}$. to classify.

Performing classification concerns domains broader that the one of crystals, quantum particles, living organisms, historical periods, languages and philosophical camps: any law of nature can also be seen as a manifestation of an objective regularity and I will try to show how the modern interpretations of symmetry allow to give a precise and, above all, coherent meaning of the terms "objective" and "regularity". It provides a rigorous ground for the meaning of universality. It also concerns the quantitative approach of inference and understanding. Some connections with the dual enquiry for emergence and reduction can 
also be drawn. Last but not least, these conceptions of symmetry shed light on a (the ?) characteristic of intelligence, namely modeling, and on an elementary process necessary for rational thinking, namely abstraction. Since,

The whole of science is nothing more than a refinement of every day thinking. It is for this reason that the critical thinking of the physicist cannot possibly be restricted to the examination of the concepts of his own specific field. He cannot proceed without considering critically a much more difficult problem, the problem of analyzing the nature of everyday thinking (Einstein, 1936, § 1, p. 349),

this feedback of the concept of symmetry from physics to science and from science to rational thinking is quite natural after all.

Twenty years ago, Edelman foresaw that there may be some possible connections between an epistemology based on norms elaborated by natural selective processes and the notion of symmetry as developed by physicists. In some sense, a part of the present work provides some consistent flesh to Edelman's "vague and utopian" (his own words) remarks:

Physics and biology will "correspond" with each other in an intimate way, certainly in the next century and possibly even sooner than that. [...] Symmetry is a stunning example of how a rationally derived mathematical argument can be applied to descriptions of nature and lead to insights of the greatest generality (Edelman, 1992, chap.20).

In this perspective, I believe that the notion of symmetry revitalises and clarifies the long-standing philosophical debate between realists and their opponents. The notions of "reality", "being", "existence" or even "self" have always been tricky because one cannot define them without introducing some tautology. In the endless controversial discussions about ontology, these notions are, at best, acknowledged as primary concepts (Einstein, 1949, pp. 669 and 673, for instance) and are, therefore, not necessarily understood or shared in the same way by all the different schools. Even more, and not only in epistemology but in the whole philosophy as well, it is quite common to find arguments for or against realism that presuppose the "existence" of what they are supposed to accept or deny. Indeed, what sense can be given to an affirmation like "reality does (not) exist"? In less caricatured sentences, like "an object is independent of the subject" or "objective facts are an illusion", the meaning of "object", "subject", "fact" or even "illusion" and the use of the verb "to be" reflect some kind of reality (otherwise we fall in a vicious circle, paved with liar-like paradoxes where illusion is itself an illusion, etc. This argument is also approved by Fine, 1996, chap. 7, § 3, first paragraph and discussed by Nozick, 2001, chap. 1).

Aside from practical ("fitted" as Darwin would have said, "convenient" as Poincaré should have preferred) everyday life, even an ultra anti-realist like a solipsist must accept the conclusion of the famous Descartes'credo "I think, therefore I am"3. Nominalists must accept the existence of equivalence classes that each word denotes. Agnostic empiricists, phenomenologists, positivists, pragmatists, idealists, etc. must deal with the existence of facts, sensations, mental representations and so on. Post-modernists of every kind use equivalence classes like historical events, social structures and groups, cultural beliefs, etc., and whether contingent or not, these classes may nevertheless become true objects of scientific studies as soon as they contribute to a rational scheme (the social or human sciences). As far as rationality is concerned-and, I would say, by definition of what rationality means - the best we can do is to remain at least non self-contradictory, in a virtuous circle so to speak. My preference in this matters inclines to distribute the notion of existence into a sort of holistic web where entities have a more or less high degree of reality according to how tightly bounded they are by coherent and logical relations. I will try to show how the four facets of symmetry, through the cardinal concept of equivalence classes, help us to gain in coherence or, at least, help us to anchor and place in this circle. Anyway, wherever we stand, an Ouroboros loop remains unavoidable because we, as intelligent subjects, are fully part of the world and

\footnotetext{
${ }^{3}$ I leave as an exercise for the reader to explain, from the hints given throughout the present article, why it is preferable to invert the implication and say "I am, therefore I think".
} 
may be, therefore, considered as objects of knowledge as well.

The two oil paintings by René Magritte entitled "La condition humaine" (the human condition) could have been chosen to illustrate the complex relation between real and its representation, but its circularity is even more admirably rendered by Maurits Cornelis Escher's lithography "Prentententoonstelling" (print gallery, see de Smit and Lenstra (2003).

Of course, I can take on safely all the self-reference aspects of the present reflections since, as being hopefully rational, they are therefore partly recursive (rational thinking must obviously be broad enough in scope to embrace the process of rational thinking itself): to play with the multiple senses of the word "reflection" and to use a metaphor borrowed from laser physics, I hope that, through this paper, some light will be produced or at least coherently amplified, like the beam in a resonant optical cavity. This somehow risky and ambitious attempt to enlighten different other fields by extrapolating some notions whose most precise meaning can only be offered by mathematics and physics was already a strategy proposed by Helmholtz in 1868, according to Cassirer (1944); the latter analyses carefully the precautions that must be taken to achieve this goal precisely with the help of the notion of symmetry.

\section{Symmetries, classifications and hierarchies}

The physical meanings of the notion of symmetry will be our guidelines throughout this essay. However, I will start by a rather formal considerations where the physical motivations remain in the background. It is only from the next sections that I will develop the physical interpretations and extend to a broader domain the matter of the present section. After the first version of this work was composed I became aware of Brading and Castellani's book. The deep connection between symmetries, classifications and groups that will be shown in $\S 2.2$ is already present in Gordon Belot $(2003, \S 5)$ and Elena Castellani $(2003, \S 2)$ 's contributions. Some considerations on the three first facets have already been proposed by van Fraassen $(1989, \S$ X.3).

\subsection{The four facets of a symmetry}

The oldest known trace of symmetry involved in a thought process are the engraved pieces of red ochre found at Blombos Cave in South Africa and are estimated to be older than 75,000 years (Henshilwood et al., 2002, fig. 2). Most likely, we will never know whether these engravings had a symbolic role or were a pure aesthetical game. However, historically, the concept of symmetry was first employed to refer to an aesthetic harmony made of unity, rhythm or balanced proportions emanating from internal or external relations Weyl (1952); Tarasov (1986); Mouchet (2013b), for instance in a sense exquisitely expressed by Charles Baudelaire in his famous poem Correspondences.

In a scientific context these associations are formulated with some mappings $T$, defined on a set $\mathscr{E}$ of elements. A symmetry associated with the set of mappings $\mathcal{T}$ encapsulates four significations that are more or less clearly distinguished in the literature:

(i). A transformation $x \mapsto T(x)$ where we retain the image $x^{T} \stackrel{\text { def }}{=} T(x)$ of an element (or a set of elements) $x$ by one mapping $T$ belonging to $\mathcal{T}$.

(ii). A comprehension: We retain all the images of $x$ obtained when applying all the mappings in $\mathcal{T}$ and collect them in the set

$$
\sigma(x) \stackrel{\text { def }}{=}\{T(x)\}_{T \in \mathcal{T}} .
$$

When applied to many (and ideally all) elements of $\mathscr{E}$ we can see the mapping $x \mapsto \sigma(x)$ as being a classification.

(iii). An invariance: Provided $\mathcal{T}$ fulfills some conditions that will be specified below, applying any mapping $T$ of $\mathcal{T}$ on any subset of $\sigma$ gives again a subset of $\sigma$. In the case of a mirror symmetry for instance, the action of $T$ on the pair $\sigma(A)=\left\{A, A^{T}\right\}$ where $A$ is a set of points of space does not modify $\sigma(A)$. 
(iv). A projection ${ }^{4}$ or symmetry breaking: Rather than dealing with the invariant sets (also called globally invariant or symmetric sets) $\sigma_{1}, \sigma_{2}, \ldots$. we retain only one element for each $\sigma$ i.e. $x_{1}, x_{2}, \ldots$ such that $x_{1} \in \sigma_{1}, x_{2} \in \sigma_{2}, \ldots$ Formally, we can see the projection as a mapping $\sigma \mapsto x$ where an $x$ is chosen to be a representative of each $\sigma$.

Projection constitutes the inverse operation of comprehension and rather than the widely acknowledged expression symmetry breaking, it should have been more precise to talk about invariance breaking.

\subsection{Symmetry as a classification tool and vice versa}

Recall that any classification requires the fundamental mathematical concept of equivalence relation: it is a binary relation, hereafter denoted by $\equiv$, between elements of a set $\mathscr{E}$ which is, by definition (Bourbaki, $1968, \S$ II.6.1 p. 113 to quote one of the paragons of structuralist mathematical books),

a) reflexive: every $x$ in $\mathscr{E}$ is related to itself, $x \equiv x$

b) symmetric ${ }^{5}$ : for all $(x, y)$ in $\mathscr{E}^{2}$, if $x \equiv y$ then $y \equiv x$

c) transitive (Euclide's first common notion): for all $(x, y, z)$ in $\mathscr{E}^{3}$ if $x \equiv y$ and $y \equiv z$ then $x \equiv z$.

From a set of mappings $\mathcal{T}$ acting on $\mathscr{E}$ we can define

$x$ to be related to $y$ if and only if there exists

a mapping $T$ in $\mathcal{T}$ such that $y=T(x)$.

Saying that this relation is reflexive means that there exists a transformation in $\mathcal{T}$ for which $x$ is invariant. A sufficient condition is of course that

a') $\mathcal{T}$ includes the identity mapping.

The symmetry property is fulfilled if

b') Every mapping is invertible and its inverse must belong to $\mathcal{T}$.

\footnotetext{
${ }^{4}$ Mathematicians would certainly prefer the term "section", keeping the term projection to exclusively qualify a mapping equal to its iterates. They would also talk of "orbit" rather than of "comprehension".

${ }^{5}$ It is not a coincidence if we recover this term here!
}

Finally, a sufficient condition to have the transitivity property of the binary relation is the closure property for $\mathcal{T}$ :

c') The composition $T_{1} \circ T_{2}$ of two mappings belonging to $\mathcal{T}$ remains in $\mathcal{T}$.

Properties a'), b'), c') are rather intuitive when using the language of transformations:

a') Doing nothing is a (somehow trivial) transformation;

b') Every transformation can be undone;

c') Making two successive transformations is a transformation.

Together with the associativity of the composition law - we always have $\left(T_{1} \circ T_{2}\right) \circ T_{3}=T_{1} \circ\left(T_{2} \circ T_{3}\right)-$ they define the set $\mathcal{T}$ as being a group. The set $\sigma(x)$ defined by (1) is called the equivalence class of $x$ and collects all the elements that are equivalent to $x$ i.e. related by the equivalence relation.

Summing up, we have seen how symmetry and classification are intimately related and why the group structure arises naturally .

Remark: It is conceptually interesting to remark that not only working with a group of transformations is sufficient for having a classification, but it is also a necessary condition in the sense that, given a classification, we can always construct an ad hoc group of transformations $\mathcal{T}$ such that (2) for the a priori given equivalence relation (see also van Fraassen, $1989, \S$ X.3). For example we can choose $\mathcal{T}$ as the group generated by all the one-to-one mappings (bijections) that are the identity everywhere but on one class. There is also the mathematical possibility of working with a set $\mathcal{T}$ that is not a group but for which (2) is still an equivalence relation. For instance take for $\mathcal{T}$ the set of all mappings $T_{\sigma}$ whose restriction to one class $\sigma$ is a bijection and that sends any element belonging to $\sigma^{\prime} \neq \sigma$ to one chosen representative element of $\sigma^{\prime}$. Except for degenerate cases where there is only one class or when all but one of them have exactly one element, $\mathcal{T}$ does not in general possess the closure property, does not contain the identity and the $T_{\sigma}$ are not invertible ${ }^{6}$. In physics I do not know any relevant examples of such a situa-

\footnotetext{
${ }^{6}$ I am grateful to Emmanuel Lesigne for helping me in clarifying these points.
} 
tion.

\section{$2.3 \quad$ Hierarchies}

The procedure of constructing new mathematical $o b$ jects as being equivalence classes is omnipresent in mathematics. The set of equivalence classes in $\mathscr{E}$ constructed from the relation $\equiv$ constitutes the so-called quotient set denoted by $\mathscr{E} / \equiv$. Just to give one fundamental example: a hierarchy of numbers can precisely be built with equivalence classes defined at each step with an appropriate equivalence relation: the rational numbers being equivalence classes of ordered pairs of integers ${ }^{7}$, the real numbers being equivalence classes of (Cauchy) sequences of rational numbers (and appear, therefore, as classes constructed from classes), the unit-modulus numbers can be seen as classes of real numbers considered as being equivalent if they differ by an integer multiple of $2 \pi$ (classes from classes from classes). More generally, we first define a new set $\mathscr{E}_{1}^{\prime}$ of elements constructed from the elements of $\mathscr{E} 1$ and then we classify them in $\mathscr{E}_{2}=\mathscr{E}_{1}^{\prime} / \equiv$ using an equivalence relation $\equiv$ defined in $\mathscr{E}_{1}^{\prime}$. Another standard example is given in Euclidian geometry: $\mathscr{E} 1$ is the set of points, $\mathscr{E}_{1}^{\prime}=\mathscr{E}_{1} \times \mathscr{E}_{1}$ is the set of ordered pairs of points and, eventually, vectors appear as equivalence classes of ordered pairs of points, two pairs of points being equivalent if and only if they form a parallelogram.

The objects formed this way gain a sort of autonomy with respect to the primitive elements from which they are built. The different operations or relations that may exist in $\mathscr{E}_{1}$ can be used to define operations in $\mathscr{E}_{2}$ provided that they do not depend on the choice of the representative elements in the classes. Therefore, computations in $\mathscr{E}_{2}$ can often (but

\footnotetext{
${ }^{7}$ By the way, in the Frege construction, even natural numbers are equivalence classes, namely the equivalence classes of sets related by one-to-one mappings (Bourbaki, 1968, §§ III.3.1 p. 157 and III.4.1 p. 166). As far as physics is concerned, I will always stay at the level of "naive" set theory without taking the subtle precautions which avoid paradoxes à la Russell, in particular by distinguishing between the so-called proper classes and sets. Perhaps, category theory should provide a more suitable framework, specially if one is akin to structural realism. As far as logical considerations are concerned, I mention that I will also take for granted the axiom of choice that allows the operation of projection.
}

not always, see the last paragraphs of $\S 6.1$ ) be done without any reference to computations at the "lower" level in $\mathscr{E}_{1}$. To keep working with the same previous example, in a $d$-dimensional space, one can add two vectors using their $2 \times d$ coordinates, not using the $2 \times 2 \times d$ numbers that encode the position of each points of the two representative pairs. Then, when constructing the upper level $\mathscr{E}_{2}$ the information that encodes the distinction between all the elements of a class at the lower level is erased. In other words the "internal position" of an element in a class has been made irrelevant or superfluous (Ismael and van Fraassen, 2003; Castellani, 2003, § 3) as far as the manipulations of the elements of $\mathscr{E}_{2}$ are concerned.

Moreover, the main motivation of constructing equivalence classes is to obtain a richer structure. One can therefore truly speak of mathematical emergence in the sense that we can draw out properties in $\mathscr{E}_{2}$ that are not relevant for $\mathscr{E}_{1}$. The emergent notions (e.g. continuity or Borel measure) concern the elements of $\mathscr{E}_{2}$ (e.g. the real numbers) rather than the elements of $\mathscr{E}_{1}$ (e.g. the integers).

According to the modern conception advocated by Klein, the characteristic properties of a multiplicity must not be defined in terms of the elements of which the multiplicity is composed, but solely in terms of the group to which the multiplicity is related.[...] The real foundation of mathematical certainty lies no longer in the elements from which mathematics starts but in the rule by which the elements are related to each other and reduced to a "unity of thought" (Cassirer, 1944, § II, pp. 7-8).

\section{Transformations}

\subsection{Active and passive physical inter- pretations of a transformation}

Let us now sew some physical flesh onto the mathematical bones I have introduced in the previous section. Let us begin with the first facet. As far as I know the distinction between passive and active interpretations of a transformation was made explicitly for the first time by Houtappel, Van Dam and Wigner $(1965, \S 2.4 \mathrm{a})$. Furthermore I will follow Fonda and 
p) Passive

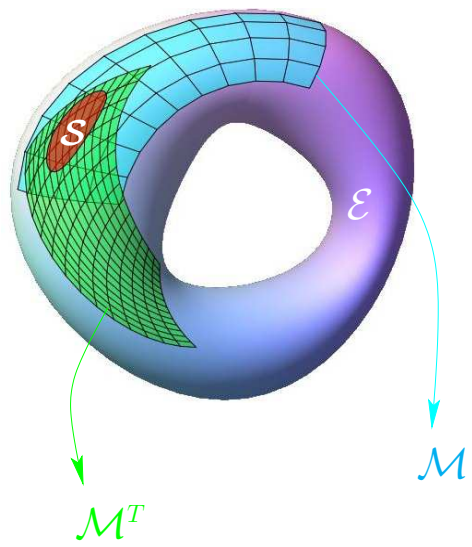

a1) Active 1

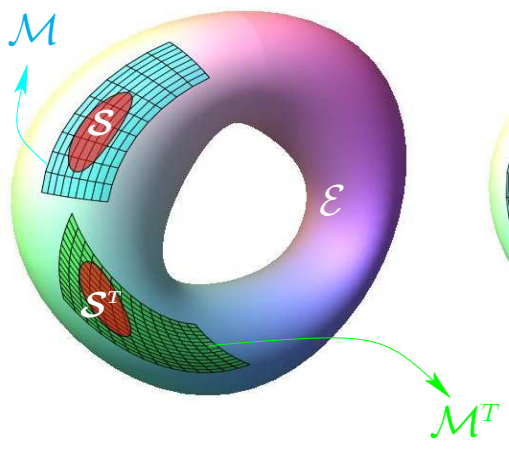

a2) Active 2

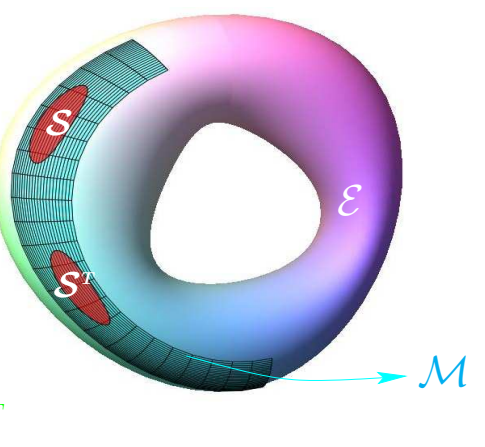

Figure 1: Schematic view of the three physical interpretations of a transformation: $p$ ) In a passive transformation the same system $\mathcal{S}$ is described by two different measurers $\mathcal{M}$ and $\mathcal{M}^{T}$. For instance, they use two kinds of measuring instruments, different gaugings or standards and two different frames of reference to map the space-time around $\mathcal{S}$. In order to give a sense to "same" and "different" used above, it is necessary to use an environment $\mathcal{E}$ as a reference. In the active points of view $\mathcal{S}$ is turned into $\mathcal{S}^{T}$ and either $\mathcal{M}$ is transformed accordingly (a1) or remains unchanged (a2).

Ghirardi $(1970, \S 1.7)$ and will consider that two active points of view can be distinguished. I will emphasize that the three physical interpretations of a transformation require the division of the universe in three parts, not two: the system $\mathcal{S}$ (the object), the measurer $\mathcal{M}$ (the subject, the observer) who describes quantitatively $\mathcal{S}$ with the help of measuring devices, an environment $\mathcal{E}$ made of everything else and that is necessary to detect whether effectively $\mathcal{S}$ or $\mathcal{M}$ have been transformed or not (figure 1). In a passive transformation, $\mathcal{S}$ remains anchored to $\mathcal{E}$ and the measurer is transformed to $\mathcal{M}^{T}$ (for instance the measuring instruments, the reference frame, the standards are changed). In the active points of view the system is changed with respect to $\mathcal{E}$ (in cosmology since, in principle, we consider $\mathcal{S}=\mathcal{E}$, the active points of view are meaningless). Unlike in the first active point of view where both $\mathcal{M}$ and $\mathcal{S}$ are changed, in the second active point, the observer $\mathcal{M}$ remains unchanged. In the latter,
[The observations are] of the same observer, using one definite language to characterize his observations (Houtappel et al., 1965, § 2.4a, p. 601).

One can find an echo of such different points of view at the mathematical level. For instance, in differential geometry, (active) transformations, like parallel transport, of geometrical objects such as vectors or forms defined on a manifold are conceptually different from (passive) coordinates changes. However, in physics (and in all empirical sciences), active and passive transformations have much stronger conceptual differences ( $\S \S 3.2$ and 3.3) as well as important operational differences ( $\S \S 3.4 .2$ and 3.5).

\subsection{Moving the boundaries}

Since $\mathcal{S}$ and $\mathcal{M}$ necessarily interact, the threshold between them can be a matter of convention: in the Michelson-Morley experiment, we may decide freely 
if the mirrors of the interferometer are included in $\mathcal{S}$ or in $\mathcal{M}$. As noted by von Neumann (1932/1955, $\S$ VI.1), this convention is linked to the choice of what facts are considered to be observed, registered and acknowledged by $\mathcal{M}$. For instance during an observation of the Sun, $\mathcal{M}$ can consider that he counts the sunspots directly or, rather, that he counts the dark spots that appear in the projection screen he uses; he can measure the degree of excitation of the retina cells of another human being looking through a telescope or look on a functional Magnetic Resonance Imaging scanner screen the activity of the corresponding brain areas during the observation. Solving the a priori ambiguity of what a "direct" observable phenomenon or a "sense datum" is consists in choosing where we place, in a whole chain of processes, the border between $\mathcal{S}$ and $\mathcal{M}$ that defines the so-called "external world" (implicitly external to $\mathcal{M}$, for a comment on this expression see Sankey, 2001, note 3). Consequently, before the interpretation in terms of correlations between data and in terms of concepts forged to cement them, this operation of cutting the chain constitutes an unavoidable task of science whose difficulty measures how remote we stand from our human sensations and from our original evolutionary-shaped abilities (the so-called intuition or common sense).

The important consequence is that, so far, we are left without criteria which would enable us to draw a non-arbitrary line between "observation" and "theory". Certainly, we will often find it convenient to draw such a to-someextent-arbitrary line; but its position will vary widely from context to context [...] But what ontological ice does a mere methodologically convenient observational-theoretical dichotomy cut?

Does an entity attain physical thinghood and/or "real existence" in one context only to lose it in another? (Maxwell, 1962, pp. 7-8).

In section $\S 4.3$ below, I will propose an attempt, based on equivalence classes, to clarify this ontological point.

Besides, quantum physics renders these issues more problematic: since no generally well-accepted quantum theory has been proposed where the unicity of the result of one measurement emerges from purely quantum processes, we still do not know how exactly the transition between quantum systems and classical measuring apparatus occurs. In the absence of a more satisfactory solution, we are forced to keep the orthodox approach where Schrödinger equation is replaced by a discontinuous transition at the very moment of measurement ${ }^{8}$. In what follows, since I will not specially be concerned by these quantum measurement issues (except in $\S 6.2$ ), I will not distinguish between the apparatus that prepares the system and the detectors. Within the very general level of the present discussion, it is not important to specify which part of the degrees of freedom belongs to $\mathcal{S}$ or to $\mathcal{M}$ and we will not be concerned by their entanglement if any (the environment $\mathcal{E}$ may have disentangled them according to the decoherence process). Furthermore, as noticed by Russell:

There has been a great deal of speculation in traditional philosophy which might have been avoided if the importance of structure, and the difficulty of getting behind it, had been realised. For example, it is often said that space and time are subjective, but they have objective counterparts; or that phenomena are subjective, but are caused by things in themselves, which must have differences inter se corresponding with the differences in the phenomena to which they give rise. Where such hypotheses are made, it is generally supposed that we can know very little about the objective counterparts. In actual fact, however, if the hypotheses as stated were correct, the objective counterparts would form a world having the same structure as the phenomenal world, and allowing us to infer from phenomena the truth of all propositions that can be stated in abstract terms and are known to be true of phenomena.[...] In short, every proposition having a communicable significance must be true

${ }^{8}$ In the last decades, significant progress have been made both theoretically and experimentally which focus on the role played by the environment during quantum evolution. Among the ocean of bibliographic references on this subject, let us mention Wheeler and Zurek (1983); Presilla et al. (1996); Breuer and Petruccione (2002); Giulini et al. (2003); Schlosshauer (2007); Barchielli and Gregoratti (2009) and their references. However, I think the central problem of quantum measurement discussed in $\S 6.2$ still remains. 
of both worlds or of neither. (Russell, 1919, chap. VI, p. 61).

I will therefore not consider the separation between $\mathcal{S}$ and $\mathcal{M}$ to be of metaphysical origin and I will not endorse any kind of genuine dualism nor pluralism but prefer a realistic (materialistic) monism: The separation between $\mathcal{S}$ and $\mathcal{E}$ (that demarcates the pertinent parameters from the irrelevant ones) together with the separation between $\mathcal{M}$ and $\mathcal{S}$ consist not only of blurred but above all of movable boundaries that must be moved to check the consistency of our line of reasoning (for an interesting proposal that trends to reinforce the conception of a unified ontology, see Clark, 2008). For instance, when the effects of the coupling to the environment are under the scope, this may be implemented by splitting $\mathcal{S}$ in two: a subsystem coupled to a bath that furnishes a reduced model of the "exterior".

Where does that crude reality, in which the experimentalist lives, end, and where does the atomistic world, in which the idea of reality is illusion and anathema begin ? There is, of course, no such border; if we are compelled to attribute reality to the ordinary things of everyday life including scientific instruments and materials used in experimenting, we cannot cease doing so for objects observable only with the help of instruments. To call these subjects real and part of the external world does not, however, commit us in any way to any definite description: a thing may be real though very different from other things we know. [...]

The boundary between the action of the subject and the reaction of the object is blurred indeed. But this does not prohibit us from using these concepts in a reasonable way. The boundary of a liquid and its vapour is also not sharp, as their atoms are permanently evaporating and condensing. Still we can speak of liquid and vapour ${ }^{9}$ (Born, 1953).

Indeed, since Samuel Johnson's refutation of Berkeley's extreme idealism-obtained by merely "striking

\footnotetext{
${ }^{9}$ No doubt that Born was also aware that one can bypass the coexistence curve and circumvent the critical point in the pressure-temperature diagram by continuously connecting the liquid phase with vapour while remaining at the macroscopic scale all the way around.
}

his foot with mighty force against a large stone, till he rebounded from it"(Boswell, 1791/2008, 6 august 1763 - - the possibility of action on the world has always been an insight of what reality means. Active and passive transformation just help to extend, precise and formalise this common sense (by the way, the use of the word "common" in this expression already bears the notion of invariance).

\subsection{Reproducibility and objectivity}

On the one hand, active points of view are at work when one wants to put to the test the reproducibility of an experiment. Galilei's ship (1632/1981, 2nd day, 317)—-see also its inspiring predecessors like Bruno's ship thought experiment (1584/1995, third dialogue) borrowed from an older argument used by Cusa (1440/1985, II.12, p. 111) - is probably the most famous and, in a historical perspective, certainly the most crucial illustration of a (first) active transformation. An example, among many others, of the second active point of view can be found in Gibbs'interpretation of a thermodynamical ensemble where we consider "a great number of independent systems" (Gibbs, 1902/2010, chap. I, p. 5 and the preface). As usual in classical pre-twentieth century tradition, the reference to any subject $\mathcal{M}$ is implicit because it is considered as being irrelevant (one noticeable exception being discussions on the Maxwell's demon).

Of course, from one experiment to another, time may have evolved. This is reflected in (or equivalent to) many transformations that occurred in the environment $\mathcal{E}$. Whether the experiment is actually reproducible or not will be a hint on the relevance of the part of the universe we have decided to consider as the system $\mathcal{S}$.

On the other hand, the passive point of view puts to the test the intersubjectivity of an observation and therefore paves the road to objectivity (see Nozick's 2001, chap. 2, p. 91). An invariance under a passive transformation reveals or defines an entity as being (at least approximately) independent of $\mathcal{M}$. To which extent this autonomy appears, and more generally the degree of reality of $\mathcal{S}$, is reflected in the nature of the group of transformations $\mathcal{T}$ for which invariance 
occurs.

Before I specify $\mathcal{T}$ in the next subsection, from what precedes we can already see how a bridge can be established between two main principles of the scientific method, reproducibility and objectivity: for this purpose, symmetry is the appropriate tool (we shall come back on this point below).

\subsection{Relevant groups of transforma- tions}

Up to now, the strong affinity between classification and transformations which was shown in $\S 2.2$ may appear of shallow interest to the physicist. Actually, one can classify anything with anything or even transform anything to anything else. When a property $P$ is given a priori "Having the same property $P$ as" defines automatically an equivalence relation and the corresponding equivalence classes for which $P$ is an invariant; reversely, if a partition of a set is given, the property $P$ can always be identified with the membership to a subset and the equivalence relation defined as "belongs to the same subset as". The transformations that are most pertinent are those that can be applied to a wide range of entities while keeping simple rules, that is, defined with less information than the set of elements they act on (we will come back in $\S 4.5 .2$ to the reduction of information provided by any classification and therefore any symmetry). A reversible transformation that turns a pumpkin into a coach, whether it concerns the three-dimensional objects or a morphing between two-dimensional images, must encapsulate the huge amount of information than encodes the pumpkin and the coach; it can hardly be applied to anything else without artificially introducing some additional ad hoc information. This is why, scientifically speaking, such a transformation is far less interesting than a rotation or a dilatation.

\subsubsection{Algebraic representations}

Indeed, the relevant groups of transformations that are considered in physics have been abstracted independently of the elements that are transformed. For instance, the usual transformations like spacetranslations or rotations have their roots in our intu- itive geometrical conception of the three-dimensional space; yet, in mathematical physics, they have now acquired the status of an "abstract" group SO(3) that can be represented in many ways: they do not apply to three-dimensional geometrical objects only but also to a huge collection of mathematical objects living in much more abstract spaces (specially the quantum states and operators). Figure 2 provides a schematic view of what an algebraic representation of an abstract group is. The theory of abstract group and their representations constitutes a whole domain of algebra and mathematical physics (among many treatises, see for instance Cornwell, 1984; Sternberg, 1994 or Jones, 1998. The special status of linear representations in quantum physics can be traced back to a theorem due to Wigner in the early 30's, see the references of Simon et al, 2008 and Mouchet 2013a.).

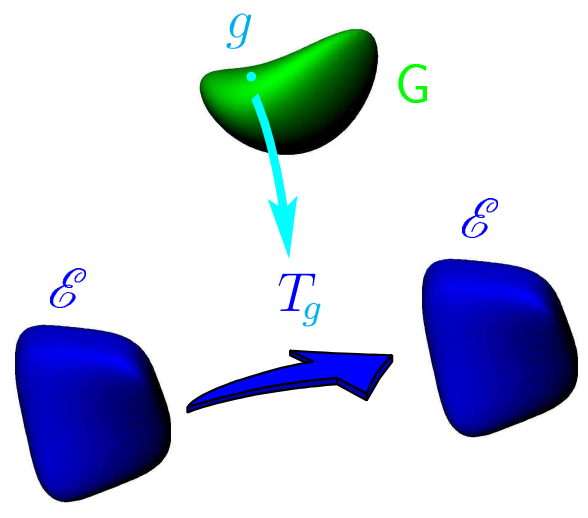

Figure 2: Schematic view of the algebraic representation of an abstract group $\mathrm{G}$ in a set of elements $\mathscr{E}$. To each element $g$ in $\mathrm{G}$ is associated a transformation $T_{g}$ acting on the elements of $\mathscr{E}$.

Many abstract symmetry groups have been shown to be relevant for the six purposes $1^{\circ}-6^{\circ}$ listed in $\S 1$. Let us direct our attention to several substantial extensions that go far beyond the set of pure spatial isometries: 
Evolution: First, the time evolution must be considered as a transformation ${ }^{10}$. This strengthens the interpretation of the transformation in terms of reproducibility since the latter concerns spacetranslations as well as time-translations. This is also in accordance with Einsteinian relativity that treats on an almost equal footing time and space.

Transformations mixing space and time: Second, in accordance with Galileo's ship argument, we can also include dynamical transformations corresponding to translations at constant speed (the transformation of spatial positions depends on time). General relativity incorporates any smooth transformation mixing space and time. Canonical formalism allows for even more general transformations involving mixture of spatio-temporal coordinates and momenta.

Exchange of particles: As suggested first by Heisenberg (1926, 1927, in particular eq. 8) and Dirac (1926), the permutations (or "substitutions") of a finite number of elements (for which the term of "group" was originally coined by Galois in 1830) appeared of crucial importance for describing quantum collective effects of indistinguishable particles. Later on, Heisenberg (1932) considered the exchange of non-identical particles, namely protons and neutrons, to describe the spectral properties of nuclei. Another important example of transformations involving changes of particles goes back to Dirac's 1930 seminal work that led to the charge conjugation symmetry (for instance, see Yang, 1994) where particles may be substituted with their antiparticles. Even though there is still no experimental evidence of its relevance, since the 1970's, the transmuting of bosons into fermions and conversely have been introduced in several particle and string models. This concept of supersymmetry is an attempt to tackle some problems raised by the Standard Model (Weinberg, 2000, $\S 24.2)$, (Binétruy, 2006, chap. 1).

\footnotetext{
${ }^{10}$ Technically, if we are to describe irreversible processes we can relax b') in $\S 2.2$ and work with a so-called semigroup.
}

Gauge transformations: Field theories that describe local interactions in condensed matter or in high energy physics, may require the introduction of some mathematical ingredients whose choice, in a finite portion of space-time, is, to a large extent, a matter of convention. A gauge transformation represents a change of convention and is, by definition of what a convention means, not expected to have any observable influence. Looking systematically for the effects of these transformations on the mathematical ingredients of a model provides sufficiently constrained guidelines to select the physically relevant descriptions. There is no need to insist any longer on the efficiency of such constructions throughout the twentieth century, from the elaboration of general relativity and Weyl's first attempts starting in 1918, to Yang and Mills (1954) non-commutative gauge model and beyond. Among the vast literature on the subject see, for instance, Moriyasu (1983) for a nice introduction.

\subsubsection{Operational differences between active and passive transformations}

All the transformations described above have been upgraded into abstract groups and can be algebraically represented within the different species of the mathematical ingredients of various physical models. However, if some transformations can be indifferently interpreted in both passive and active point of view, this is far from being the case for all of them.

Precisely because gauge transformations fulfill their constructive role when they have no detectable consequences, they can only be interpreted as passive transformations (Brading and Brown, 2004, and its references). The same situation occurs in classical mechanics: after a relevant canonical transformation that interweaves them, the dynamical variables may loose the empirical interpretation they had before being transformed (say, the positions and velocities of the planets when blended into action-angle variables). Therefore, such transformations appear clearly as a change of description of the dynamics rather than an active transformation of the system.

Examples of active (resp. passive) transformations 
that cannot naturally be considered from the passive (resp. active) point of view are given by transformations associated with an exchange, addition or subtraction of some constituents of the system (resp. the measuring device). If we are ready to accept a permutation between quantum particles as a candidate for being in $\mathcal{T}$, why should we not consider also the replacement of a spring in a mechanical apparatus or the modification of the wavelength of a probe? Even though, these kinds of transformations are never (as far as I know) considered when talking about symmetry, this is precisely invariance with respect to them, as being discussed in $\S 5$ below, that allows eventually to establish stable and universal laws. In practise, when attempting to reproduce an old or a remote experiment, the experimental set up is rarely moved but rather completely rebuilt from new materials.

\subsection{Perception of space-time trans- formations: following Henri Poincaré's intuitions}

In fact, a way of understanding which mathematical transformations can be interpreted both passively and actively was presented more a century ago by Poincaré (1895; 1903, § 5) when analysing carefully our intuition of three-dimensional space (his considerations can be applied to a large extent to the fourdimensional space-time). He introduced a distinction that Einstein would sum up four decades after him:

Poincaré has justly emphasized the fact that we distinguish two kinds of alterations of the bodily object, "changes of state" and "changes of position." The latter, he remarked, are alterations which we can reverse by arbitrary motions of our bodies (Einstein, 1936, § 2, pp. 354$355)$.

However, what does not appear clearly in this report (but was criticised by Einstein elsewhere, for instance in his 1949 , p. 677 reply) is that, according to Poincaré, this is a matter of definition or convention:

Thence comes a new distinction among external changes: those which may be so corrected we call changes of position; and the others, changes of state (Poincaré, 1903, § 5, I underline).

For Poincaré, the possible compensation of an active transformation by a passive one is therefore intimately linked with our perception of space.

For a being completely immovable there would be neither space nor geometry; in vain would exterior objects be displaced about him, the variations which these displacements would make in his impressions would not be attributed by this being to changes in position, but to simple changes of state; this being would have no means of distinguishing these two sorts of changes, and this distinction, fundamental for us, would have no meaning for him (Poincaré, $1903, \S 5)$.

However, following Galileo's conception of uniform speed, Poincaré was aware that transformations could not be considered absolutely and, as soon as 1902, he clearly formulated in chap. V, $\S \mathrm{V}$, of Science and Hypothesis a "relativity law". The transformations must be anchored somewhere: this is precisely the main reason why we must introduce the environment $\mathcal{E}$ as a background and that Einstein $(1936, \S 2$, p. 354) would call a "bodily object". Otherwise, the first active point of view would be undetectable and the distinction between the passive and the second active point of view would be meaningless. Extending an old argument going back to Laplace (according to Jammer, 1994, chap. 5, pp. 168-169), Poincaré not only discusses the case of a uniform expansion of the universe but also considers any continuous change of coordinates:

But more; worlds will be indistinguishable not only if they are equal or similar, that is, if we can pass from one to the other by changing the axes of coordinates, or by changing the scale to which lengths are referred; but they will still be indistinguishable if we can pass from one to the other by any 'point transformation' whatever. [...] The relativity of space is not ordinarily understood in so broad a sense; it is thus, however, that it would be proper to understand it (Poincaré, 1903, § 1). 
Poincaré considers that only topological structuresbeing unaffected by homeomorphisms - are physically relevant. For him the choice of differential structure, in particular through the metric, is a matter of convention similar to a choice of units or a choice of language. The imprint of curvature in the sum of the angles of a triangle or on the ratio between the perimeter of a circle and its radius can always be rectified empirically.

Think of a material circle, measure its radius and circumference, and see if the ratio of the two lengths is equal to $\pi$. What have we done? We have made an experiment on the properties of the matter with which this roundness has been realized, and of which the measure we used is made (Poincaré, 1952, chap. V, $\S 2)$.

and also

The same question may also be asked in another way. If Lobatschewsky's geometry is true, the parallax of a very distant star will be finite. If Riemann's is true, it will be negative. These are the results which seem within the reach of experiment, and it is hoped that astronomical observations may enable us to decide between the two geometries. But what we call a straight line in astronomy is simply the path of a ray of light. If, therefore, we were to discover negative parallaxes, or to prove that all parallaxes are higher than a certain limit, we should have a choice between two conclusions: we could give up Euclidean geometry, or modify the laws of optics, and suppose that light is not rigorously propagated in a straight line. It is needless to add that every one would look upon this solution as the more advantageous. Euclidean geometry, therefore, has nothing to fear from fresh experiments (Poincaré, 1891).

Albeit historical development of the theory of gravitation has refuted Poincaré's opinion that

Euclidean geometry is, and will remain, the most convenient: 1st, because it is the simplest, and it is not so only because of our mental habits or because of the kind of direct intuition that we have of Euclidean space; it is the simplest in itself, just as a polynomial of the first degree is simpler than a polynomial of the second degree; 2nd, because it sufficiently agrees with the properties of natural solids, those bodies which we can compare and measure by means of our senses (Poincaré, 1891).

when gravitation was formulated as a gauge theory by Utiyama (1956), then working either with Euclidian/Minkowskian or Riemannian geometry appeared indeed to be a conventional choice. See Carnap (1966, chaps. 15-17), Stump (1991) or Hacyan (2009) for a more detailed discussion on these issues. (Even if we put aside the topological properties of space-time, like worm-holes at large scales or any kind of topological defect that may have physical observable consequences which would definitely rule out Minkowskian geometry, some general arguments on causality still favour a curved space-time interpretation, Penrose (1980)).

Even if we keep sticking to Riemannian geometry, we can remark that a confusion remains that obscures the debate between realists and conventionalists (or their relativist post-modern successors). A representation (here a choice of one coordinate map) is always, obviously, a matter of convention (it fixes a gauge); however, when quotienting all the equivalent descriptions obtained one from each other by a transformation (the change of coordinates), we can abstract geometrical objects with intrinsic properties (the Riemannian manifold itself and the tensors defined on it). Formally from a set of quantities indexed by coordinates labels, say $v^{\mu}$, whose values change when changing the coordinates from $\left(x^{\mu}\right)_{\mu}$ to $\left(x^{\mu^{T}}\right)_{\mu}$,

$$
v^{\mu T}=\frac{\partial x^{\mu T}}{\partial x^{\nu}} v^{\nu},
$$

an abstract object can be defined, the tangent vector $v$, which is independent of the precise choice of coordinates. While the coordinates (3) are said to be co-variant under the (passive) transformation that changes the coordinates, the vector $v$ remains insensitive to it. Besides, to emphasize the intrinsic character of the construction, rather than starting from the transformation rule (3), a vector at a point $x$ is preferably defined in differential geometry, as being an equivalence class of tangent differential curves 
on the manifold passing through $x$ (Choquet-Bruhat and DeWitt-Morette, 1982, chap, III, B.1, pp. 120 121). This geometrisation procedure was the core of Klein's Erlanger program (1893, see Cassirer, 1944, for a historical account on the connections between a group of geometrical transformations, perception, invariance and objectivity. See also the excerpts given above in $\S 2.3$ and below in $\S 5$ ) and one of the fathers of the modern notion of symmetry was already aware of its physical connection with objectivity (for a recent survey, see Kosso, 2003, § 2):

Without claiming to give a mechanically applicable criterion, our description bears out the essential fact that objectivity is an issue decidable on the ground of experience only.[...] Whereas the philosophical question of objectivity is not easy to answer in a clear and definite fashion, we know exactly what the adequate mathematical concepts are for the formulation of this idea.[...] The pure mathematician will say: Given a group $\mathrm{G}$ of transformations in a field of symbols, a geometry is established by agreeing to study, and consider as objective, only such relations in that field as are invariant under the transformations of G (Weyl, 1949, $\S 13$, pp. 72 and 77$)$.

Perhaps the philosophically most relevant feature of modern science is the emergence of abstract symbolic structures as the hard core of objectivity behind - as Eddington puts itthe colourful tale of the subjective storyteller mind (Weyl, 1949, Appendix B, § 1, p. 237).

and also

But I preferred the name of automorphisms [... ], defining them with Leibniz as those transformations which leave the structure of space unchanged.[...]

We found that objectivity means invariance with respect to the group of automorphisms. Reality may not always give a clear answer to the question what the actual group of automorphisms is, and for the purpose of some investigations it may be quite useful to replace it by a wider group. For instance in plane geometry we may be interested only in such relations [that] are invariant under parallel or central projections; this is the origin of affine and projective geometry. The mathematician will prepare for all such eventualities by posing the general problem, how for a given group of transformations to find its invariants (invariant relations, invariant quantities, etc.), and by solving it for the more important special groups - whether these groups are known or are not known to be the groups of automorphisms for certain fields suggested by nature. This is what Felix Klein called "a geometry" in the abstract sense. A geometry, Klein said, is defined by a group of transformations, and investigates everything that is invariant under the transformations of this given group (Weyl, 1952, chap. II, p. 42 and chap. IV, pp. 132-133).

Poincaré's conventionalism far from being antithetic to objectivity (see Giedymin, 1982, 1992) appears to be a necessary condition for the latter:

Now the possibility of translation implies the existence of an invariant. To translate is precisely to disengage this invariant.[...]

What is objective must be common to many minds and consequently transmissible from one to the other, and this transmission can only come about by [a] "discourse" [...] we are even forced to conclude: no discourse, no objectivity. [...]

Now what is science? I have explained [above], it is before all a classification, a manner of bringing together facts which appearances separate, though they were bound together by some natural and hidden kinship. Science, in other words, is a system of relations. Now we have just said, it is in the relations alone that objectivity must be sought; it would be vain to seek it in beings considered as isolated from one another.

To say that science cannot have objective value since it teaches us only relations, this is to reason backwards, since, precisely, it is relations alone which can be regarded as objective.

External object, for instance, for which the word object was invented, are really objects and not fleeting and fugitive appearances, because they are not only groups of sensations, but groups cemented by a constant bond. It is this bond, and this bond alone, which is the object in itself, and this bond is a relation (Poincaré, 1902, $\S \S 4$ and 6, I underline). 
The concept of symmetry allows to give a quantitative meaning to the terms used in this remarkable text: "Relations" and "classification" have already been discussed in $\S 2.2$. "Translation" (not taken here to synonymous of a shift!) appears to be a passive transformation in the sense considered in section $\S 3.1$, and its relation with objectivity has been emphasized in $\S 3.3$; "object" being considered as an equivalence class, an "invariant". Whereas "discourse" (we have seen above that Houtappel et al. also use the term "language") corresponds to a choice of convention (but, of course, Poincaré is far from endorsing nominalism, see $\S 4$ of his 1902 reflexions), a coordinate chart in differential geometry or more generally a representative of an equivalence class, that "breaks" the symmetry (or, rather, the invariance).

\section{Comprehension and classifica- tion}

Poincaré's last remark on the importance of classification and its role in identifying invariants leads us to focus on the second facet of the concept of symmetry.

Comprehension is an old scholastic notion and it is not by chance that the same word is used to mean the act of comprehending and the act of understanding. The latter requires to find analogies ${ }^{11}$, correlations, regularities between a priori distinct entities or phenomena; these connections are the most precisely expressed by equivalence relations (incidentally, relatives constitute the fourth Aristotelian category; Aristotle 1938, § IV, pp. 16-17) which are synonymous, as we have seen, of classification and therefore of unification. The common qualities shared by the elements of one class are the invariants and allow to define them by intension rather than by extension. The duality between a class and its elements reactivates, to use a terminology with some medieval taste, the old opposition between universals and individuals.

\footnotetext{
${ }^{11}$ See Kant (1781/1998, I, 2nd part Transcendendal logic, 1st division, book II, chap. II, § III.3, Analogies of experience) and Cournot (1851/1956, chap. IV § 49).
}

\subsection{From taxonomy to predictability}

We do not have to probe very deeply to acknowledge the major role of taxonomy in science. Far from being a purely descriptive tool, sorting things out is above all relevant when it reveals some objective properties, as Poincaré explains in the last quotation given above. In biology, for instance, the hierarchical classification initiated by Carl Linnaeus which were originally based on phenotype only, allowed to reveal some correlations that could be explained within the general scheme of the theory of evolution ${ }^{12}$. The degree of similarity between some physical or even behavioural characteristics of living organisms have been a relevant indicator of the chronology of the divergence between species. Even before the genetic distance was even conceived and before phylogenetics, these correlations could be associated (through correlations of correlations, so to speak) to the history of our planet (climate fluctuations, atmosphere composition, continental drifts, etc).

Another example is provided by the appropriate way of classifying chemical elements which led Mendeleev to some successful predictions on the properties of elements that were yet unknown in 1869 . Without knowing the atomic structure (at an epoch where only few physicists accepted the existence of atoms), this periodic classification exhibits some universal characteristics that were not undermined by the subsequent improvements of the physical theories and the numerous models of the atoms that came with them (first classical, like Kelvin's vortex atoms in 1867 and later on, of course, the quantum models).

During the second half of the twentieth century, symmetry has strengthened its classificatory and predictive role. Originated in the 1930's by Wignerwho received in 1963 the Nobel price in physics precisely "for his contributions to the theory of the atomic nucleus and the elementary particles, particularly through the discovery and application of fundamental symmetry principles"- our modern classification of quantum particles, whether there are supposed to be elementary or not, relies on the classifica-

\footnotetext{
${ }^{12}$ More generally, the role played by symmetries is of the greatest importance in all hierarchy-based philosophies, particularly in Cournot's 1861, book IV, chap. XIV, § 514.
} 
tion of the (linear) algebraic representations (in the sense defined in $\S 3.4 .1$ ) of groups like the Poincaré group (Wigner's philosophical reflections on symmetry are collected in his 1995 book. For a modern presentation of technical details see Weinberg, 1995, chap. 2). Unlike taxonomy of macroscopic objects, the classification of quantum particles relies on a very few parameters only, like the mass, the electric charge, the spin. The reason is that in quantum physics only very few observables are compatible with all the others (technically, those associated with operators that commute with all the others, which are associated with the so-called superselection rules). Otherwise, an observed property is generically completely erased by the measurement of an incompatible observable: quantum particles have no history nor accidents and quantum field theory naturally incorporates the indistinguishability of the particles belonging to the same class. Anyway, the hadron classification leading to the prediction of the $\Omega^{-}$or the prediction of the existence of particles like the $W^{ \pm}, Z^{0}$ or the Higgs boson in the Standard Model are all built on symmetry arguments (more specifically on the basis of a symmetry breaking argument). These provide another illustration, like in Mendeleev's contribution, that the possibility of classification is a signature of an objective reality, the latter being taken in the sense given by Poincaré in the last quotation. In the same time, this classification often reveals a substructure (the periodic table reflects the atomic structure, the "eightfold way" to classify the hadrons reflects the quark structure and maybe the quark and leptons classification reflects a still unknown subworld).

\subsection{Inference and the problem of change}

Since any time-translation can be considered as a particular case of symmetry transformation $(\S 3.4)$, the foresight skills conferred by evolution equations (whether classical or quantum) appear as the most important aspects of the predictive power provided by symmetry. Obviously, this ability of anticipation covers fields much broader than science. There is no need to solve differential equations to successfully forecast that the sun will rise tomorrow morning. Af- ter having closed your eyes, you know with almost certainty that you will find the words you are currently reading in the same order once you look again on this page. There is no miracle here (Putnam, 1975, chap. 4, p. 73), just a consistency for rational thinking, a raison d'être: no thoughts, no language could be possible if we were unable to discern some "constant bond" as Poincaré wrote. These stable properties (at least for a moment long enough to be noticeable) reveal what we call the existence of things, building up together what we call the real world (including ourselves, see for instance Mach, 1906/1976, chap. XXV, SS 14, 17). A macroscopic object like a cherry can be considered as an equivalence class associated with properties (shape, taste, colour, size, etc.) that remain invariant despite the change of light, the endless adsorption or desorption of molecules (active transformations in the sense explained in § 3.1) or the (passive) transformations of the spatial position of the measurer or of his neural states. As remarked by Heisenberg (1958, chap. IV) or Popper (1969, chap. 5, $\S$ IX), this "problem of change" (or of becoming) has already been settled by pre-Athenian philosophers among which Heraclitus and Parmenides, who proposed the two most extreme solutions along the whole continuous spectrum of possible answers.

We will come back in section 5 to the notion of invariance, which constitutes the third facet of symmetry. However, at this stage, we can usefully notice that symmetry as a predictive tool provides an intermediate support for the inductive/deductive inference. Induction, as a reasoning from a part to a whole is formally described by the operation $x \mapsto \sigma(x)$ while deduction, processing from general to particular instances, offers a second look at the fourth facet of symmetry, formally described by the operation $\sigma \mapsto x$.

\subsection{Stability and abstraction}

As analysed by Taine 150 years ago, any intelligent being is characterised by his ability of constructing a representation of the world (see also Cournot, 1851/1956, chap. VII, § 109):

Thus, every normal sensation corresponds to some external fact which it transcribes with 
greater or less approximation, and whose internal substitute it is. By this correspondence, internal events agree with external, and sensations, which are the elements of our ideas, find themselves naturally and beforehand adjusted to things, which adjustment will, further on, enable our ideas to be in conformity with things, and consequently true. On the other hand, we have seen that images are substitutes for sensations, past, future, or possible, that individual names are substitutes for images and sensations momentarily absent, that the more simple general names are substitutes for images and impossible sensations, that the more complex general names are substitutes for other names, and so on. It seems then that nature has undertaken to provide in us representatives of her events, and has effected her purpose in the most economical way. She has provided, first, the sensation which translates the fact with more or less precision and delicacy; then, the surviving sensation capable of indefinite revival, that is to say, the image, which repeats the sensation, and consequently translates the fact itself; then, the name, a sensation or image of a particular kind, which, by virtue of its acquired properties, represents the general character of many similar facts, and replaces the impossible images and sensations which would be necessary to translate this isolated character. By means of this correspondence, of this repetition, and this replacement, external facts, present, past, future, special, general, simple, or complex, have their internal representatives, and this mental representative is always the same internal event, more or less compounded, repeated, and disguised. (Taine, 1872, end of book III, chap. II, $\S \mathrm{V}$, p. 141-142).

This correspondence between the world and a mental representation ${ }^{13}$ is necessarily partial not only because a brain, as a tiny part of the universe, can only

\footnotetext{
${ }^{13}$ Not surprisingly there is an abundant literature that discusses the congruence between objects and beliefs since it determines the nature of truth. As old as western philosophy itself, this subject is omnipresent in logic, in linguistic, in psychology, in jurisprudence, in epistemology etc. If we are ready to move the boundaries as suggested in $\S 3.2$, the traditional opposition between coherence and correspondence theory of truth should be softened (see also the discussion in Weyl, 1949 , $\S 17$, p. 117).
}

encode far less information than the universe itself (James, 1880), but also for the sake of the efficiency that enforces its own stability as an organised structure, as needed by Darwinian competitive selection (Popper, 1979, § 2.16; Dawkins, 1976, chap. 4; Dennett, 1996, chap. 4). The processing of informationmemorising, learning, anticipating - by any intelligent entity cannot be done without a discrimination between relevant and irrelevant parameters (by the way, the famous Wisconsin card sorting test allows neuropsychologists to quantify the adoption of classification rules and measure its flexibility by trial and error, see for instance $\S 6.5$, p. 412 of Baars and Gage, 2010).

Ireneo Funes, the character dreamt by Jorge Luis Borges, whose memory was rigorously infallible,

was almost incapable of general, platonic ideas. It was not only difficult for him to understand that the generic term dog embraced so many unlike specimens of differing sizes and different forms; he was disturbed by the fact that a dog at three-fourteen (seen in profile) should have the same name as the dog at three-fifteen (seen from the front) (Borges, 1942, Funes, the memorious, p. 114).

He who cannot forget cannot generalise; he who cannot generalise cannot think. Classification appears therefore as a fundamental thought process and hence has been the subject of a wide field of research in cognitive sciences where the synonym "categorisation" is usually preferred (Rosch, 1978, Smith, 1998; Murphy, 2002, chap. 7; Machery, 2009, chap. 6, for recent surveys of this matter). Abstraction, forging concepts, using a language, constructing models and eventually building up theories are variations on the same theme, namely, the second facet of symmetry. Observation itself appears to be an act of abstraction - actually the lowest level of abstraction - inconceivable without entities, the socalled observable entities, that are indeed concepts (Duhem, 1906/1954, part 2, chap. IV, $\S \S 1,2$ and chap. VI, § 1) emerging when pruning the information out of a bunch of data extracted from a magma of occurrences. Following Hanson (1958, chap. 1, $\S \mathrm{C})$ and talking about theory-laden observations is 
therefore awkward, unless we differentiate between multiple levels of abstraction, because it supposes a theory-independent core to be loaded and, thus, this leads immediately to a self-contradiction since this core appears to be an abstract notion in itself. In that sense, in my criticism of empiricism, I am going further than Hanson and according to Sellars (1956) I would rather prefer talking about the "myth of the given".

\footnotetext{
In sum, facts are facts, and if it happens that they satisfy a prediction, this is not an effect of our free activity. There is no precise frontier between the fact in the rough and the scientific fact; it can only be said that such an enunciation of fact is more crude or, on the contrary, more scientific than such another (Poincaré, $1902, \S 3)$.
}

De facto, the degree of precision of any classification depends on the criteria we retain. Fruits can be classified according to the kind of desserts you can make with or according to the genome of its plant. Stars can be grouped in constellations or in galaxies (Cournot, 1851/1956, chap. XI, § 161). The criteria are always moving, are often arbitrary or contingent and maybe difficult to define without referring to the category itself: the most efficient way to explain to a child what a cherry is remains to show him several examples of this fruit, not to give a definition (Poincaré, 1903, § 4). This intuitive approach is all the more natural than brains are instinctively inclined to think in terms of associations that nurture its creativity (Hadamard, 1954; Weil, 1960, for instance). Unlike computers, brains are analogue and selectionist rather that digital and instructionist devices (Popper, 1978, p. 346; Edelman 1992, specially the critical postscript, Dehaene, 1997, specially the chap. 9). The reluctance of being classified offered by certain elements requires reflection, testifies the weakness of our comprehension and gives evidence of the rich variety and of the impermanence of the world. A thing or a word can come with many variations of significations, without which there cannot be nuances, ambiguities, metaphors and even poetry.

But unlike art, rational thinking and, consequently, its most elaborated form that science represents, tends to sharpen and perennialise the boundaries of the equivalence classes; it brings out criteria of truth beyond cultural barriers and socio-historical context. This strategy allows to reach provinces far away from our natural (i.e. adequate from an evolutionary point of view) narrow county and, as we have seen in the previous section, to make predictions (Llinás, 2001, specially chap. 2). I have already mentioned ( $\S 2.3)$ the hierarchy of numbers in mathematics; it extends the concept of numbers much further than the primitive mental representations of integers, genetically encoded with a broad brush in highest mammal brains (Dehaene, 1997; Butterworth, 1999). In physics, the both prominent frameworks of general relativity and quantum theory constitute charts at scales considerably much wider than our everyday life environment. If we adopt the adequation I propose between existence and equivalence classes, the questions of knowing if we invent or rather discover these provinces, if symmetry unveils or sculptures reality, appear to be unessential (see also Margolis and Laurence, 2007); it is a matter of taste depending on where we prefer to observe the virtuous circle (for a particularly interesting examination of this old subject, namely the "reality of abstractions", see Cournot, 1851/1956, chap. XI, specially the arguments in $\S \S 152$ and 155). In addition, this orientation also allows to soften the question of the adequacy between the mathematical world and the physical world: if we merge these two worlds then the effectiveness of mathematics does not appear as an unreasonable miracle (Wigner, 1960) but it is still true that it is puzzling to see how far this fitness can spread.

\subsection{Different levels of Darwinian se- lection}

The segmentation of the world in equivalence symmetry classes, and the drawing of more or less fuzzy boundaries between them (the most fundamental being the partition in $\mathcal{M}, \mathcal{S}$ and $\mathcal{E}$ as explained in $\S \S 3.1$ and 3.2 ), can be seen as a selection process. Jacques Hadamard (1954, first note of chap. III, p. 29) recalls that the Latin original meaning of intelligence is "selecting among" (inter-legere) and echoes the 
Poincaré's statement:

What, in fact, is mathematical discovery? It does not consist in making new combinations with mathematical entities that are already known. That can be done by any one, and the combinations that can be so formed would be infinite in number, and the greater part of them would be absolutely devoid of interest. Discovery consists precisely in not constructing useless combinations, but in constructing those that are useful, which are an infinitely small minority. Discovery is discernment, selection (Poincaré, 1908).

It is of course Darwin's major contribution to have shown how differential survival governs the evolution of living species. This natural selective stabilisation is a (non-linear) mechanism at work in many other dynamical systems made of different entities whose populations are susceptible of attenuation and amplification (via reproduction, replication, combinations, etc.). It would be too long a digression, and beyond my competence, to examine the subtle distinctions of how Darwinian selection is implemented in so various fields as immunology, cognitive sciences, computer science, sociobiology and culture. Even in evolutionary biology, the details of the picture are still objects of scientific controversies among specialists like Richard Dawkins, Stephen Jay Gould, William D. Hamilton, Richard Lewontin, John Maynard Smith, Edward Osborne Wilson, to name some popular contemporary figures among many others (Segerstråle, 2000; Shanahan, 2004).

With the philosophical bet of building a conception of the world in a coherent ontology the stakes are too high for the philosophy of physics alone ; it must as well rely on a Darwinian like naturalization that makes some norms to emerge. To remain within the scope of the present paper, I just wanted to point out that the formation of some equivalence classes I denoted by $\sigma$ can be seen as a natural process à la Darwin not only when $\sigma$ denotes a living species, a pool of genes or of memes (elements of material or immaterial culture, including knowledge, Auger, 1952; Dawkins, 1976, chap. 11), but also if we adopt a neurobiologist point of view. Any class $\sigma$, considered as an object i.e. a part of the physical world (a predator, a cherry, a pixel, an atom) may be represented in terms of some neural configuration distributed in several intertwined areas of the brain. Since more abstract concepts $(\sqrt{2}$, a quantum phase, entropy, a blue tiger, Prometheus, beauty, freedom, etc.) and, more generally, ideas are also encoded in brains via the neural material support, the insurmountable difficulty of delimiting what reality is can be understood at the neurophysiological level (the recursive character appears here through consciousness, i.e. when the model of the world that characterises intelligence includes a representation of itself). Some concepts (a cherry, the Moon, an electron, a number, Kepler's law, a rotation, yourself) are strongly reinforced and stabilised by being intricately bound within a large hierarchy, including observations or sense-data, while some others (a blue tiger, a tachyon, chess rules, the pumpkin-coach transformation, Zeus), being less firmly established, have a smaller degree of reality. As any practitioner of quantum physics knows it (even before the modulus of some electronic wave-function in quantum corrals has been imaged by scanning measurement), the concept of a quantum state generally refers to a more real entity that the toy concept of a solid sphere made of gold with a radius of $1 \mathrm{~km}$.

Recent investigations in neuroscience seem to give a neural basis of an old suggestion made by James (1880) and to confirm the existence of Darwinian (epigenetic) selective mechanisms between various pre-representations generated by combinatorial games throughout neuron networks. Many selectionist processes may be distinguished that are characterised by transformations of neural states at different time-scales; compared to the fast dynamics of, say, decision making, the neural Darwinism involved in learning and memorisation may concern slower developments of neural circuitry, starting before birth soon after conception (dendritic and axonal generation or regression, synaptic reinforcement or inhibition, etc.). The "survival of the fittest" representation would be determined by complex tests of evaluation, reentrance and reinforcements procedures (driven by pleasure and pain for instance) that involve the internal dynamics of the brain as well as its coupling to its external environment (includ- 
ing other brains). We still know very few about how this dynamics is physiologically implemented; however, it shares many common points with selectionism at another level, namely the evolution of scientific knowledge. Since the first proposals of an "evolutionary epistemology" by Campbell (1959, 1960 and their numerous references that anticipated the subject even if James is not mentioned) and Popper (1979) (see also Toulmin, 1967, 1972; Campbell, 1974), this approach has known many outgrowths (Radnitzky and Bartley, 1987; Hull, 2001). For a recent review of the neural selection introduced by Changeux et al. (1973); Edelman (1978) called neural Darwinism by the latter (1987), see Seth and Baars (2005), Platek, Keenan and Shackelford (2007) and references therein. For a less specialised presentation see, for instance, Changeux $(1985 ; 2002)$ or Edelman (2006). The emergence of a pattern of successes from an "overwhelming background of failures" (Fine, 1996, chap. 7, $\S 1$, note 5) in science is therefore explained in an analogous way as the formation of stable and viable combinations of genes from the overwhelming possibilities of deadly mutations (as far as the cumulative progress of knowledge is concerned, we have not been able to make up the memetic analogue of such an efficient process like sexuality for generating reliable combinations. However, in these matters, from the association of ideas, both at the mental level and at the human collaboration level, we are closer to the memetic equivalent of meiosis than to agamogenesis, see however the conservative trends described by Toulmin, 1967, § VI).

\subsection{Symmetry-assisted research simplicity}

\subsubsection{Simplicity as a general evaluation against underdetermination}

Most obviously, one may fit a given discrete set of registered events with many different laws (Cournot, 1851/1956, chap. IV). Still, this so-called underdetermination, acknowledged under one form or another since Epicurus (in the Letter to Pythocles, see Laertius, 1925, chap. X, mainly $\S \S 86-88$ ) and carefully examined by Hume (1758/1999), still abundantly dis- cussed in epistemological debates on induction (Laudan, 1990; Psillos, 1999, chap. 8). Notwithstanding, underdetermination appears to be less serious than some philosophers think it is, provided that we take into account the notion of simplicity as one of the selection criterion (see Poincaré's 1891 citations given above together with his 1900a article; Cournot, 1851/1956, chap. IV, § 41; Mach, 1906/1976, §§ X.15, XXV.6,7 Jeffreys, 1931, chap. IV; Popper, 1959, chap. VII and 1969, chap. 1, point (6) of the appendix, chap. 10, § XVIII; Reichenbach 1938, § 42). Following the same lines of thought drawn above, the aim of this section is to suggest how the second facet of symmetry is at work in this Darwinian-like selective operation.

The problem of simplicity is of central importance for the epistemology of the natural sciences. Since the concept of simplicity appears to be so inaccessible to objective formulation, it has been attempted to reduce it to that of probability, which has already been incorporated to a large extent into mathematical thought (Weyl, 1949, § 21, pp. 155-156).

Here we join back the aesthetical appeal of a symmetry we briefly recalled in the very beginning of $\S 2.1$. The harmony of a theory, the beauty of an argument or the elegance of an experiment are frequently evoked by scientists (Fresnel, 1818; Poincaré, 1908; Russell, 1917, chap. IV; Hardy, 1940; Weyl, 1949, § 21; Heisenberg, 1974, chap. XIII; Penrose, 1974; Lipscomb, 1980; Yang, 1980; Dirac, 1963, 1982; Chandrasekhar 1987; Weinberg, 1992, chap. VI; Changeux 2012), some of them considering it as a constructive principle if not a criterion of truth. For a curious attempt to quantify aesthetics using a, somehow vague, notion of complexity see Birkhoff (1933); for a more serious and interesting study of the role of aesthetics in science see McAllister (1996) where Haldane's text below is partially quoted (chapter 7 provides an actualised discussion on simplicity, see also the diverse contributions on this subject in Zellner et al., 2001). One reason of this attractiveness is the gain in simplicity, a parsimony of descriptive means with respect to the wide range of their scope (see for instance Hamilton, 1861, 
Appendix I(A), fifth theory, p. 580 $)^{14}$.

In scientific thought we adopt the simplest theory which will explain all the facts under consideration and enable us to predict new facts of the same kind. The catch in this criterion lies in the word 'simplest'. It is really an aesthetic canon such as we find implicit in our criticisms of poetry or painting. The layman finds such a law as $\frac{\partial x}{\partial t}=\kappa \frac{\partial^{2} x}{\partial y^{2}}$ much less simple than 'it oozes', of which it is the mathematical statement. The physicist reverses this judgement, and his statement is certainly the more fruitful of the two, so far as prediction is concerned. It is, however, a statement about something very unfamiliar to the plain man, namely, the rate of change of a rate of change. Now, scientific aesthetic prefers simple but precise statements about unfamiliar things to vaguer statements about well-known things. And this preference is justified by practical success (Haldane, 1927, Science and Theology as Art Forms, p. 227).

Broadening the scope of a theory and reducing the number of laws and primitive concepts are two faces of a single coin. The tension between these two antagonistic tendencies is the mainspring of the progress of scientific knowledge. Focusing on just one of those opposite poles leads to ridiculous positions: on the one hand, if we consider only very few particular events, we can explain their occurrence by arbitrary simple or rather simplistic laws and concepts (this is one of the main characteristic of superstitions and pseudo-sciences) and the complication comes from the multiplication of such ad hoc explanations as the number of observations increases (see for instance Popper, 1969, chap. 1, § I point (7) and its appendix or Worrall, 1989, p. 114). On the other hand, as Feynman puts it with his inimitable style (Feynman et al., 1970, vol. II,§ 25-6), from any physical law (not necessarily discovered yet!) labelled by $l$, once written in the (dimensionless) form $Q_{l}=0$, we can always introduce the physical concept of "unworldliness" associated with this law, namely $U_{l} \stackrel{\text { def }}{=}\left|Q_{l}\right|$ where $|\cdot|$ de-

\footnotetext{
${ }^{14}$ Even before the "least action" or other variational principles were acknowledged, a parsimony principle is already present in Galileo's work ("Nature does not act by means of many things when it can do so by means of a few", 1632, 2nd day).
}

notes the modulus or the norm, and write the "equation of everything" as

$$
U=0
$$

where the total unworldliness is given by $U=\sum_{l} U_{l}$. Of course this trick to hide the complexity under the simplest conceivable equation remains sterile because of the artificial and shallow interpretation of $U$. As Feynman explains it, this stratagem is not motivated by any symmetry arguments: because it does not take into account any transformation rules, its nature is very different from the grouping of the scalar quantities $v^{\mu}$ into one compact geometrical object $v$ (see $\S 3.5$ p. 14).

However, since Pythagoras, we can find many attempts based on aesthetic and symmetric grounds that have failed to provide correct explanations. Up to his very last years Kepler did not want to abandon his cosmological model based on Platonic solids though he acknowledges, in many notes of the 1621 edition of the Mysterium Cosmographicum, that his beautiful construction is wrong (25 years after the first edition and after the discovery of the three laws; there is some kind of irony here since Kepler himself contributed to undermine the symmetrical appeal of the "harmony of perfect spheres" with the first law which manifests the breaking of the rotational invariance through one individual elliptic orbit). If the absence of any experimental evidence in the LHC experiments is confirmed, it may be that supersymmetry should be abandonned, at least in its simplest form.

\subsubsection{Symmetry as a pruning tool}

One of the major flaws of the attempts to define physical complexity/simplicity from algorithmic information theory is that it relies on information compression without any loss (Delahaye, 1999; Li and Vitányi, 2008, for an upgraded account on this vivid field and for the original references). In fact, to gain in simplicity we must be allowed to irreversibly cut some superfluous hypothesis or initial conditions with an Ockham's razor. Moreover, we have seen in $\S 4.3$ that information erasing is essential because classifi- 
cation is primordial, ubiquitous and necessary ${ }^{15}$. We cannot avoid working with some equivalence classes, some of which we nonetheless take as primitive entities by not considering the distinct properties that allow to discriminate its elements, ones from the others. By quotienting a set in equivalence classes, the second facet of symmetry is more a mean of elimination than a mean of compression. Here we stick to the original meaning of abstraction, considered as a synonymous of removal (Cournot 1851/1956, chap. XI, $\S \S 147-149$ discusses several processes of such a disentanglement). Algorithmic reduction appears only to be a special case of labelling each class by one privileged element $x^{*}$ (specifically, one of the shortest) and, then, the algorithmic depth of $x$ quantifies the transformation $T$ that maps $x^{*}$ to $x=T\left(x^{*}\right)$. However, this algorithmic interpretation can hardly embrace the physical world without substantial new foundations; because of continuity and randomness, whether they are fundamental or just convenient, most, if not all, physical transformations cannot be reduced in a discrete sequence of binary operations. As far as I know, nobody has been able to provide the algorithmic complexity or the algorithmic depth of an electron.

Here we join back the thermodynamical (or Shannon) conception of information, where entropy measures the loss of information that occurs when considering a statistical ensemble rather than the individual systems (or messages, if we are concerned with information transmission). The latter is described in terms of a huge number of variables $\varphi$ (say, the microscopical variables like the positions and the velocities of classical atoms) while the former is characterised by few variables $\Phi$ (say, the average velocity of the atoms, their total energy, etc.). The art of statistical physics consists precisely in identifying quantitatively the relevant variables $\Phi$ that

\footnotetext{
${ }^{15} \mathrm{~A}$ parallel point of view is proposed by Landauer (see, for instance his 1967 paper and the collection of papers on information erasure edited by Leff and Rex, 2003, specially chap. 4) and leads to a modest realism different from the one proposed here. Landauer adopts a strong ontological difference between mathematical entities and reality by denying the existence of $\pi$, for instance (Landauer, 1999, p. 65). The proposal I prefer to defend here is that what $\pi$ and a cherry designate differ one from the other by their degree of reality on a continuous scale.
}

allow a tractable account of which $\varphi$ 's are compatible with $\Phi$ (reduction) and how $\Phi$ can be obtained from $\varphi$ (emergence). We often speak of macroscopic variables (resp. of a macrostate) when considering $\Phi$ (resp. the ensemble), by contrast to the microscopic variables (resp. micro or pure state) that $\operatorname{refer}(\mathrm{s})$ to one element. This is often justified because the spatial extension usually allows to intuitively discriminate both systems (fluid/molecules, galaxies/stars, heavy nucleus/nucleons, etc.). It may also happen, when studying dynamical systems, that we may take advantage of a separation of time scales between a fast evolution of $\varphi$ and a slow, secular, evolution of $\Phi$. If we want to circumvent the favouritism of space and/or time, it is preferable to see the mapping $\varphi \mapsto \Phi$ as the erasing (the decimation as Kadanoff would say, 2000, chaps. 13 and 14) of irrelevant degrees of freedom. This is the quantitative formulation of the classification $x \mapsto \sigma$ when the elements $x$ (resp. $\sigma$ ) can be labelled with the numerical variables $\varphi$ (resp. $\Phi)$; the irrelevant parameters being those, discrete and/or continuous, that allow to label the different transformations $T$ of $\mathcal{T}$.

One given statistical ensemble contains microstates that differ one from the other by transformations of $\varphi$ compatible with the macroscopic constraints fixed by $\Phi$. Building out the effective model consists in coarse-averaging that is, integrating out, say within the partition function, the variables that allow to discriminate the microstates (in quantum statistical physics this represents the computation of partial traces of the density operator) and retaining the dependence in $\Phi$ only. Some of the individual properties of the particles - typically the ones involved in the interactions like the charges but not their individual energy for instance - are unaveraged and remain as such among the $\Phi$. How some properties of the effective system depend on such variables reflects the degree of universality of the construction. It is one of the greatest achievements of statistical theory to have indeed exhibited some quantities (the electromagnetic radiation emitted by a black-body, the critical exponents) that depends only on the grossest features of the system (the dimension of space, etc.) and neither on chemical composition nor on the precise form of the interactions, as does the crit- 
ical temperature of a second order phase transition. Less universal but much simpler examples of $\Phi$ are the pressure of a fluid or the absorption coefficient $\alpha$ of a material. Both have their value depend on the details of the microscopic interactions but, surprisingly, they can be used to characterise a wide variety of substances whose microscopic structures strongly differ one from the other (for instance $\alpha$ is involved in the Beer-Lambert law to describe the light transmission through a liquid, a gas, a plastic, a glass, a diamond). The emergence of universality due to the law of large numbers was already noticed by (Langevin, 1923, chap IV, p. 191) and, before him, by (Poincaré, $1911, \S$ IV) who talked in this case of the insensitivity to large differences in the initial conditions.

More generally, beyond statistical physics, from all the preceding considerations presented in this section, we can understand that picking up a small number of relevant variables $\Phi$ out of a wide collection of $\varphi$ remains an indispensable mechanism of any cognitive process.

\subsection{Emergence}

The last line of thought conveys us into the core of the debate about reduction/emergence issues (Bedau and Humphreys, 2008). Since, recently, there have been many discussions on these matters (Batterman, 2010; Butterfield, 2010), I will not venture too long into this wide realm (see also for instance Castellani, 2002). At the level of abstraction I adopt in this article, I formally take up the stance that emergence stands for constructing the variables $\Phi$ describing $\sigma$ from the variables $\varphi$ describing $x$. Reduction, appears to be the opposite operation $\sigma \mapsto x$ (the fourth facet of symmetry, I will have to say more about it below). Thus, we have identified the two dual elementary mechanisms from which are built the hierarchies that structure the physical world and shape rational thinking. We have encountered an example of mathematical emergence in $\S 2.3$. In $\S 4.5 .2$, I have sketchily recalled how statistical physics, within the frame of renormalisation theory, offers what is perhaps the most precise quantitative illustration of the rearrangement and elimination of the degrees of freedoms that allow to establish the relation between $x$ and $\sigma$. In every-day thinking, this is intuitively accomplished by the categorisation capacity of what we call the common-sense that make a cherry or a dog to emerge from a bunch of interweaved organic molecules (or cells if we want to add some intermediate structure).

Identifying what properties are relevant or convenient at one level and establishing quantitatively their connection with the properties at other levels remains a formidable challenge, the nub of scientific research I would say. There is no general method following well-established instructions to increase our comprehension (again, our individual minds and the web of our socially interconnected minds are rather more selectionist than instructionist, more Darwinian than Lamarkian so to speak). This is obvious when following the reductionist direction; however, it is not less true when climbing up the stairs in the other way. Digging out the emergent properties by picking up a small number of relevant variables among a wide collection can seldom be done explicitly. Above, I used the word "art" to qualify statistical physics not, of course, to deny the standards of rational thinking which I fully endorse, nor to indicate a direction of increasing beauty ${ }^{16}$, but precisely to emphasize that there were no general rule of how to elaborate an effective theory, and even more to compute (specially when infinities are to be dealt with), the $\Phi$ from the $\varphi$. The large number of appropriate parameters $\Phi$ and the difficulties to establish protocols of their measurements mainly explains why medicine, social sciences, psychology, economics, ecology, agriculture, and even the technical aspects of cooking etc. are less exact (i.e. predictive with less precision) than more quantitatively supported branches of science like chemistry or physics. The objects of investigation of the latters are not only relatively much simpler but also the statistical fluctuations are much more reduced by the huge number of elements in a sample (as far as I know it is only in physics that

\footnotetext{
${ }^{16}$ As Weinberg (1992, chap. VI) writes it, general relativity may appear more beautiful than Newton's theory though involving far more quantities, namely the infinitely many degrees of freedom of the gravitational field. In Newtonian theory, the gravitational field does not have a proper dynamics that can be made, even partially, independent of the dynamics of matter.
} 
self-averaging quantities can be exhibited). Even in every-day rational thinking, the variables $\Phi$ remain mostly qualitative rather than quantitative and naive attempts of their formalisation often appear as dangerous oversimplifications.

\section{Invariance}

In the introduction of $\S 3.4$, I have shown how the second facet of symmetry allows to identify classification with property attribution. I have explained in $\S 4.2$ why these two equal cognitive capacities, inherent to any kind of measurement (including perception), can be viewed as a stabilisation.

The general concept of group pre-exists in our minds, at least potentially. It is imposed on us not as a form of our sensitiveness, but as a form of our understanding ; (Poincaré, 1895, conclusions).

A sine qua non condition of intelligibility is that some physical properties must remain unchanged under transformations like some space or time translations, rotations, etc. I precise "some" because this stability necessarily exists in a finite portion of spacetime only: The correlated bundle of attributes that allow to characterise Borges'dog lasts more than one minute but certainly no more than 30 years. The norm and direction of the terrestrial acceleration $\vec{g}$ remain approximately uniform at laboratory scales but change according to where we are on Earth; moreover, of course, it is only meaningful in the immediate neighbourhood of the Earth, which in its turn has a finite space-time extension. Some quantum properties like those of the kaon $K^{ \pm}$last $10^{-8} \mathrm{~s}$ while others like the electron charge (more precisely the fine-structure "constant") seem not to vary on cosmological scales more than one part in $10^{6}$ (as far as we can safely measure up to now $)^{17}$.

\footnotetext{
${ }^{17}$ By the way, while we construct the equivalence classes we consider as physical objects, whether we prefer to implement or not invariance under some time-translations remains a matter of convenience. Both approaches are admissible and broadly equivalent in a non-relativistic approach but, of course, the time extension cannot be avoided as soon as relativity is taken into account since then, simultaneity becomes a relative
}

It is the greatest merit of Noether's theorems to provide a locally conserved quantity associated with each independent one-dimensional continuous family of transformations under which the functional driving the dynamics (more precisely, the action together with appropriate boundary conditions) is invariant. I will not devote more attention to this specific aspect of invariance since the implications and the interpretations of Noether's work have already been scrutinised elsewhere (Kosmann-Schwarzbach, 2010; Brading and Brown, 2004, notably). What I want to propose in $\S 5.1$ is a more general perspective where invariance, viewed as the third facet of symmetry, concerns not only formulations of variational principles but, in fact, any law of science. Then, in $\S \S 5.2$ and 5.3 , I will open this interpretation to even broader epistemic considerations.

This rudimentary tendency toward "objectification" reappears in conceptual, in particular mathematical, thought, where it is developed far beyond its primitive stage. When we determine the size of an object by measurement, it is owing to such "objectification" that we succeed in transcending the accidental limits of our bodily organisation. It enables that elimination of "anthropomorphic elements" which is, according to Planck, the proper task of scientific natural knowledge. To geometrical invariants have to be added physical and chemical constants. It is in these terms that we formulate the "existence" of physical objects, the objective properties of things.[...] Hering speaks [...] the language of the scientist, i.e., of realism. He assumes the empirical reality of the objects about which our senses have to inform us. But a critical analysis of knowledge must go farther. Such an analysis reveals that the "possibility of the object" depends upon the formation of certain invariants in the flux of sense-impressions, no matter whether these be invariants of perception or of geometrical thought, or of physical theory. The positing of something endowed with objective existence and nature depends on the formation of constants of the kinds men-

property. The philosophical numerous discussions between endurantists and perdurantists seem therefore neither obscure nor very interesting once it is illuminated by physics arguments (see, for instance Butterfield's 2005 point of view and the references provided there). 
tioned. It is, then, inadequate to describe perception as the mere mirroring in consciousness of the objective conditions of things. The truth is that the search for constancy, the tendency toward certain invariants, constitutes a characteristic feature and immanent function of perception. This function is as much a condition of perception of objective existence as it is a condition of objective knowledge (Cassirer, 1944, end of $\S$ III, pp. 20-21).

Born is also very clear:

I think the idea of invariant is the clue to a rational concept of reality, not only in physics but in every aspect of the world.

[...] not every concept from the domain of scientific constructs has the character of a real thing, but only those which are invariant in regard to the transformation involved. [...]

This power of the mind to neglect the differences of sense impressions and to be aware only of their invariant features seems to me the most impressive fact of our mental structure.[...]

Thus we apply analysis to construct what is permanent in the flux of phenomena, the invariants. Invariants are the concepts of which science speaks in the same way as ordinary language speaks of "things," and which it provides with names as if they were ordinary things (Born, 1953).

I perfectly agree except that I would nuance and would prefer to say that "not every concept shares the same degree of reality" because, as I will recall below, invariance of a "real thing" always appears to be an approximation.

\subsection{Science laws as quantitative man- ifestations of invariance}

Whatever school of thinking one belongs to, whatever status one attributes to a science law ${ }^{18}$, it ought

\footnotetext{
${ }^{18}$ Among the overwhelming literature on this subject, I should mention van Fraassen's 1989 influential attempt to deny laws of nature while, strangely enough, keeping the notion of symmetry. If I am not mistaken, what van Fraassen criticises is the dogmatic conceptions of laws, specially when we forget that their quantitative formulations should come with their conditions of validity.
}

to be uncontroversial that most, if not all, science laws yields to relations between quantities $x$ that can always be written as ${ }^{19}$

$$
\mathscr{R}(x)=0 .
$$

Frequently, these relations are written in the most explicit form

$$
q=\mathscr{Q}(y)
$$

by privileging among the $x$ 's some quantities, namely $q$, against others, namely $y$. I use here two different fonts to discriminate between mappings, here $\mathscr{Q}$ and $\mathscr{R}$, and the physical quantities, here $x, y$ and $q$. These mathematical relations (often taken by many to be synonymous of the laws themselves) have a physical meaning only when they are embedded in a theory where the quantities $x$ or $(q, y)$, have been related to observations, via a model incorporating more or less intermediate other quantities. This does not imply that all the $x$ 's should be uniquely determined by measurements; some of them may be unobservable, superfluous or irrelevant. Moreover, when talking about laws, relations (5) or (6) should not be confused with definitions. "All ravens are black" can represent either a partial definition of what a raven is or a law if the class of ravens and the blackness have been defined by other ways. Both status are mutually exclusive but remain a matter of choice as we keep these instances in a coherent network of significations. History of science is full of examples where theorems/laws/synthetic judgements have switched to axioms/definitions/analytic judgements and vice versa. See, for instance, the discussion by Weyl (1949, $\S 17$, p. 114) on the status of the electric field $\vec{E}$ in the equation giving the electrostatic force $\vec{F}=e \vec{E}$; or Feynman's 1970, § 12-1 explication of the meaning of the force as it appears in Newton's second law $\vec{F}=m \vec{a}$.

A transformation $T$ that is performed on the variables $x$ defines a transformation of (5) according to

\footnotetext{
${ }^{19}$ Inequalities, like Heisenberg's or the second law of thermodynamics can also be turned to this form with the help of Heaviside step function. The mathematical relation between the $x$ 's is of course not unique. For instance, $E-m c^{2}=0$ and $\exp \left(1-E / m c^{2}\right)-1=0$ are strictly equivalent expressions. However, here also, a principle of parsimony should help to select a convenient form.
} 
the rules

$$
\mathscr{R}^{T}\left(x^{T}\right)=\mathscr{R}\left(x\left(x^{T}\right)\right)
$$

where, in the right hand side, $x$ are considered as functions of the transformed variables $x^{T}$. Then, in the second case, the transformed function $\mathscr{Q}^{T}$ is defined by

$$
q^{T}=\mathscr{Q}^{T}\left(y^{T}\right)
$$

Relation (5) will be said invariant if the same relation exists between the transformed quantities $x^{T}$ :

$$
\mathscr{R}(x)=0 \quad \Longleftrightarrow \quad \mathscr{R}\left(x^{T}\right)=0 .
$$

If $q$ stands for just one scalar quantity with respect to $T$ i.e. that by definition $q^{T}=q$ (this not an essential point, the argument can be adapted if we were to work with non trivial linear or even non-linear algebraic representations of $T$ ), then invariance of (6) is formally equivalent to the equality between the two functions $\mathscr{Q}^{T}$ and $\mathscr{Q}$, i.e. for all $y$

$$
\mathscr{Q}^{T}(y)=\mathscr{Q}(y)
$$

In some circumstances, using the tools developed in catastrophe theory, the structural stability of relations (5) or (6) is reasonably guaranteed (Poston and Stewart, 1978; Arnold, 1984; Demazure, 2000, to retain three remarkable introductions to this subject). Anyway, the general notion of invariance I want to consider here comes closer to Poincaré's conception that pervades in his 1902 quotation in $\S$ 3.5. Let us just mention two simple examples, involving neither integrals nor derivatives and only scalars, not tensors, not fields, nor operators: Kepler's third law connecting the orbital period $P$ of a planet whose semi-major axis length is $a$ :

$$
\frac{P^{2}}{a^{3}}-\frac{4 \pi^{2}}{G M}=0
$$

( $M$ is the mass of the Sun, $G$ stands for the gravitational constant); and the equation giving the period $P$ of the harmonic oscillations of a mass $m$ attached to an ideal spring of strength $\kappa$ :

$$
P=2 \pi \sqrt{\frac{m}{\kappa}} .
$$

First, these equations manifest some invariance with respect to the usual transformations that constitute the Galilean group and that can be both interpreted passively or actively. Indeed, equation (11) remains as correct now as it was 350 years ago. Equation (12) is valid in any laboratory on Earth, even if embarked on a boat with constant speed. However, the raison d'être of a law is an even broader notion of invariance: if we actively transform the mass-spring system by varying the mass $m \mapsto m^{T}$ and/or substituting the spring by a different one $\kappa \mapsto \kappa^{T}$, we may wonder if the transformed period of the oscillator will still be given by the same law

$$
P^{T}=2 \pi \sqrt{\frac{m^{T}}{\kappa^{T}}} .
$$

Kepler's third law is valuable not only because it is still accurate for Mars since Kepler's century, but also because we can keep the same relation if we jump from Mars to Venus (then, formally, $a$ and $P$ are transformed into $a^{T}$ and $P^{T}$ but neither $M$ nor $G$ are altered) or if we change the centre of attraction by considering the orbital periods of the Galilean moons around Jupiter (now, formally $M \mapsto M^{T}$ as well). Even more, $G$ is qualified as a genuine universal constant precisely because in a given system of units (that encapsulates connections with other quantities conventionally considered as standard ones), its value remains unchanged when performing the previous transformations.

These considerations can be transposed without difficulties to any field mature enough to be described by reasonably quantitative laws; for instance to Mendel's statistical laws of heredity, to equations governing some Darwinian dynamics or to Kleiber metabolic law (for refinements on this latter subject, see for instance Brown and West, 2000). As we recalled in the introduction, it is in modern quantum theories of fields and in relativity that invariance has been first set up as a constructive principle. What is postulated is the a priori invariance of $q$ (a Lagrangian density, an action, a partition function, a transition amplitude, etc.) with respect to some transformations or, in other words, the independence of $q$ with respect to some quantities among the $y$ 's that appear to be superfluous (the absolute spatial 
position, the gauge fields, etc.). It happens that these symmetry principles may impose, on the choice of $\mathscr{Q}$, constraints stringent enough to select the models drastically and compel specific dynamics on the interactions (gauge symmetries dictate the properties of the photon or the graviton for instance). This line of pursuit yields to a feeling of deep inevitability (Weinberg, 1992, chap. VI), despite the criticisms of "the reason which accounts for uniformity in nature" made by van Fraassen (1989, chap. II, § 2).

\subsection{Modest truth and humble univer- sality}

I hasten to add that relations like (5) or (6) remain always conditionally true. Ideally, in mathematics, theorems should be formulated with all their hypothesis. In the same way, when faced with empirical verification and with numerical tests, laws should come with some error bounds, whether of statistical or systematical origin, even if their conditions of validity can never all be explicited Ullmo (1969, § II.B). In other words, as far as predictivity is at stake, we should include an evaluation of expected risk or loss.

If we look at any particular law, we may be certain in advance that it can only be approximative. It is, in fact, deduced from experimental verifications, and these verifications were and could be only approximate. We should always expect that more precise measurements will oblige us to add new terms to our formulas this is what has happened, for instance, in the case of Marriotte's law.

Moreover the statement of any law is necessarily incomplete. This enunciation should comprise the enumeration of all the antecedents in virtue of which a given consequent can happen. I should first describe all the conditions of the experiment to be made and the law would then be stated: If all the conditions are fulfilled, the phenomenon will happen.

But we shall be sure of not having forgotten any of these conditions only when we shall have described the state of the entire universe at the instant $t$; all the parts of this universe may, in fact, exercise an influence more or less great on the phenomenon which must happen at the instant $t+\mathrm{d} t$.

Then as one can never be certain of not having forgotten some essential condition, it can not be said: If such and such conditions are realized, such a phenomenon will occur; it can only be said: If such and such conditions are realized, it is probable that such a phenomenon will occur, very nearly (Poincaré, 1902, § 5).

Indeed (see $\S 3.2$ ), any modelisation requires an abstraction of the relevant quantities describing the system $\mathcal{S}$ from the irrelevant degrees of freedom that are swept away in the environment $\mathcal{E}$. The mass $m$ of the planet does not appear in Kepler's third law but this is true up to terms of order $m / M$. The latter may be not negligible any longer if we want to describe a binary star. If damping is at issue, among the infinitely many external parameters that have not been considered in the spring-mass model, the air viscosity must be incorporated in the right hand side of (12) together with a reinterpretation of $P$ as a pseudo-period. For transformations such that $m^{T}$ is too large, we may also quit the linear regime; then, the period will depend on the initial amplitude (but still not on the position of the moons of Jupiter!).

One famous criticism against a realistic conception of science is the pessimistic (meta-)induction according to which the falsity of the past scientific theories should lead us to conclude that present theories are not reliable either (Putnam, 1978; Laudan, 1981, 1984). However, this argument is fallacious (another reason was recently given by Lewis, 2001) because it presupposes for science a too ambitious, unreachable and eventually meaningless goal. Following Zénon, the Renaissance protagonist of Marguerite Yourcenar's novel The Abyss,

I have refrained from making an idol of truth, preferring to leave to it its more modest name of exactitude (Yourcenar, 1976, p. 123, A conversation in Innsbruck).

Indeed, realism should remain "modest" (Bricmont and Sokal, 2001) since the reduction of information requested by any modelisation implies that the truth of a statement, of a prediction, can be conditional only. The most we can require from a science law is to come with the knowledge of its limitation and hope- 
fully with a quantitative control of the errors; but, of course, this can be achieved once the theory is mature enough and/or embedded in a wider one as a socalled effective theory. If we are to use a softer metainduction argument (qualifying it as pessimistic or optimistic is a litmus test to determine whether you think that the science cup is half empty or half full), if we follow the lessons of history of science and consider that there cannot be but effective theories. The "Dreams of a final theory" (Weinberg, 1992) remain an act of faith not far from the idealistic, therefore unrealistic, desire of Platonic truth (for a fair and recent clarification on the subject see Castellani, 2002). Nevertheless we can argue, for instance, that Newtonian mechanics remains universally true for macroscopic objects with small enough masses and velocities. Albeit we know relativity and quantum physics, computation of the location and the dates of eclipses will still be correctly done within the old classical framework; the main improvements on the precision of the predictions and retrodictions of eclipses are expected to come from a refinement of the planetary models (possibly with post-newtonian amendments but certainly not with full general relativity and even less quantum corrections) and are known to be limited in time by the chaotic character of the manybody (classical) problem. In that sense, Pythagoras' theorem or Kepler's law have not been undermined by the development of non-euclidean geometry, general relativity or quantum physics.

The thing essential is that there are points on which all those acquainted with the experiments made can reach accord.

The question is to know whether this accord will be durable and whether it will persist for our successors. It may be asked whether the unions that the science of today makes will be confirmed by the science of tomorrow. To affirm that it will be so we can not invoke any $a$ priori reason; but this is a question of fact, and science has already lived long enough for us to be able to find out by asking its history whether the edifices it builds stand the test of time, or whether they are only ephemeral constructions.

Now what do we see? At the first blush it seems to us that the theories last only a day and that ruins upon ruins accumulate. Today the theories are born, tomorrow they are the fashion, the day after tomorrow they are classic, the fourth day they are superannuated, and the fifth they are forgotten. But if we look more closely, we see that what thus succumb are the theories, properly so called, those which pretend to teach us what things are. But there is in them something which usually survives. If one of them has taught us a true relation, this relation is definitively acquired, and it will be found again under a new disguise in the other theories which will successively come to reign in place of the old (Poincaré, 1902, § 6) ${ }^{20}$.

For the same essential reasons that lead us to estimate the degree of reality with a continuous balance, I prefer not to consider truth to be a binary quantity. I will not follow Kuhn, specially chap. IX, X, XII, XIII neither Feyerabend on their emblematic fields: talking about incommensurability between two theories is often an overstatement and I would keep the term to qualify logically contradictory assertions or dogmatic ideologies only (to play with the words, recall that etymologically 'symmetry' is the Greek form of the latin 'commensurable'). Thus the notion of scientific revolution is just a convenient tool, at best, to simplify the vivid evolutionary river basin of science made of bifurcations as well as fusions of many streams. As usual, there is no fundamental cleavage but a (seemingly continuous) gradation that separates entities different in degree but not in nature.

Science laws including their domain of validity appear to be the far most stable pieces of knowledge; they give evidence for reality or, rather, these hard core knowledge have the highest degree of reality. This is why not only any scientific knowledge must be falsifiable in the Popperian sense, but it definitely must be falsified. It is only when a law fails that we can estimate its domain of validity; then, we can expect to get a broader law that will encapsulate, protect and therefore perpetuate the first one.

\subsection{Epistemic invariance}

Extending the notion of symmetry transformations outside the usual corral of space-time transformations gives us a bird's-eye view of what science laws represent. In particular, the perspective I proposed above

\footnotetext{
${ }^{20}$ See also Poincaré's quotation given by Worrall (1989, p. 103).
} 
allows to reinforce the connection between invariance and objectivity (see also Nozick's 2001 general reflexions on this point where a non-binary degree of truth and objectiveness is proposed). Of course we have preserved elements of human culture much older than Pythagoras' theorem, Archimedes' or Kepler's laws but, unlike science laws (Sokal and Bricmont, 1998, the intermezzo), most are crucially dependent on their contextual signification and appear to be fragments partially detached from their contemporary cultural background; we appreciate their value with criteria that are now completely different from the ones that were in vogue at those ancient times. It is precisely a cornerstone of the structuralism method applied to various fields of human science (including linguistic and anthropology) to find out structural invariants that are shared by the whole of mankind (and even further, the functional invariants that are shared by substrates of variable structure, likewise the brain functions in regards to the neuroanatomical organisation, see Changeux, 2002, § VI.7):

Any classification is superior to chaos and even a classification at the level of sensible properties is a step towards rational ordering. It is legitimate, in classifying fruits into relatively heavy and relatively light, to begin by separating the apples from the pears even though shape, colour and taste are unconnected with weight and volume. This is because the larger apples are easier to distinguish from the smaller if the apples are not still mixed with fruit of different features. This example already shows that classification has its advantages even at the level of aesthetic perception (Lévi-Strauss, 1968, chap. 1).

At a philosophical level, as suggested by Weyl in the conclusion of his philosophical book, despite the apparently irreducible divisions between numerous philosophical schools, we can identify some common denominators (the invariants) that reveal some sort of unity beyond what is often-but of course not always - merely a semantic variation, a matter of subjective preference or a pure intellectual game.

The more I look into the philosophical literature the more I am impressed with the general agreement regarding the most essential insights of natural philosophy as it is found among all those who approach the problems seriously and with a free and independent mind rather than in the light of traditional schemes - or if not agreement then at least a common direction in their development. Whether one talks about space in the language of phenomenology like Husserl or 'physiologically' like Helmholtz is less important, in view of their substantial concordance, than it appears to the 'standpoint philosophers' who swear by set formulae (Weyl, 1949, end of $\S 23 . \mathrm{D}$, p. 216).

I hope to have shown that symmetry is actually a good candidate for being such an invariant as far as rationalists are concerned, among whom, at least, Poincaré's heirs like Worrall (1989) (see also more recent approaches like the one proposed by Esfeld, 2006) and his successors - who belong to the multiple subdivisions of the structural realist branch (Bokulich and Bokulich, 2010)—but, maybe, also the supporters of Fine's natural ontological attitude (Fine, 1996, chap. 7) or even those who prefer to endorse Van Fraassen's constructive empiricism.

It would certainly be too presumptuous to paraphrase Dawkins'expression 1976, chap. 3 and consider the scientific laws to be "immortal" meme complexes; however, it is sure that they constitute the far most stable ones. History shows that they have been able to resist the most dramatic memocides (like the multiple destructions of the library of Alexandria) or the most insidious eumemisms (like the selective copy of texts during the western Middle Ages that almost completely stemmed the flow of greek materialist philosophy initiated by the ancient atomists Leucippus and Democritus, then adapted by Epicurus). Recall,

If, in some cataclysm, all of scientific knowledge were to be destroyed, and only one sentence passed on to the next generations of creatures, what statement would contain the most information in the fewest words? I believe it is the atomic hypothesis (or the atomic fact, or whatever you wish to call it) that all things are made of atoms-little particles that move around in perpetual motion, attracting 
each other when they are a little distance apart, but repelling upon being squeezed into one another. In that one sentence, you will see, there is an enormous amount of information about the world, if just a little imagination and thinking are applied (Feynman et al., 1970, vol I. $\S 1-2)$.

\section{Projection}

\subsection{Invariance breaking}

We have already encountered the fourth facet of symmetry in various places.

From an epistemic point of view, this is precisely the reductionist strategy. The choice of one element in an equivalence class constitutes the basic operation of decomposing a class in its constituents: real numbers enclose rational numbers, the class of fruits comprises cherries, the class of dogs includes Borges'dog, atoms contain electrons, one-phonon states are built from condensed atomic states, etc. Together with the elaboration of effective models and the identification of emergent properties discussed in $\S 4.6$, reduction ties the hierarchical web of interlaced concepts.

From a logical point of view, we talk about deduction in the sense that we infer some properties on an $x$ as soon as it is identified as an element of class $\sigma$ (e.g., "Hume is mortal because humans are"). In that sense, $x$ may be considered as a prototype (I prefer to use this word, following Rosch, 1978, rather than the too pejorative "stereotype" or the too much connoted by idealistic flavour "archetype") of the class and a systematic choice of one representative in each class can be used to label the classes, specially if a natural (canonical) rule can be proposed (for instance, rational numbers are represented and can even safely be identified to a fraction of coprime integers). Here, when talking about the representation of $\sigma$ by $x$, both acceptions b) and c) in footnote 2 fit in this scheme.

There is an alternative way to interpret the projection operation $\sigma \mapsto x$. The identification of one element $x$ among all the others is possible only if we are able to attribute properties to $x$ that are not characteristic of $\sigma$ but, rather, whose values allow to discriminate its elements. The differences are encapsulated in the set $\mathcal{T}$ of transformations connecting two elements. Because of these distinctive properties, the global invariance of $\sigma$ under $\mathcal{T}$ is broken since $x \neq x^{T}$ in general. As acknowledged by Pierre Curie in a famous paper, asymmetry is essential to characterise the physical phenomena:

What is necessary is that certain symmetry elements do not exist ${ }^{21}$. Asymmetry is what creates the phenomenon (Curie, 1894, § IV, p. 400).

For more modern variations on this theme, see the interesting essay by Morrison (1995, On Broken symmetries. pp. 99-114).

In practise, the manipulation of concepts often requires the use of one of its representative. For instance, in theoretical physics computing scalar products requires the choice of a representation of each vector by introducing a specific basis. In differential geometry a coordinate chart is most often required to proceed with numerical computations. When making measurements, some frame, gauge and units must be used and, more generally, a measurer $\mathcal{M}$ maps some part of the universe that includes the physical system at issue (see $\S 3.1$ and figure 1). Let us hand over to Weyl

A typical example of this is furnished by a body whose solid shape constitutes itself as the common source of its various perspective views (Weyl, 1949, § 17, p. 113).

and Born, who uses an analogy with the different elliptical shapes of the projected shadows of one circular cardboard (this simple but acute example is already in Cournot, 1851/1956, chap. XIII, § 198),

This root of the matter is a very simple logical distinction, which seems to be obvious to anybody not biased by a solipsistic metaphysics; namely this: that often a measurable quantity is not a property of a thing, but a property of its relation to other things. [...] Most

\footnotetext{
${ }^{21}$ I translate literally the original French. Rosen and Copié's 1982 English translation interprets the words " $n$ 'existent pas" in a more appropriate and careful way and propose "are missing" instead of "do not exist".
} 
measurements in physics are not directly concerned with the thing which interest us, but with some kind of projection, this word taken in the widest possible sense. The expression coordinate or component can also be so used. The projection (the shadow in our example) is defined in relation to a system of reference (the walls, on which the shadow may be thrown) (Born, 1953, p. 143).

In any case some superfluous variables are introduced that mask the objectivity by breaking the invariance of the equivalence classes. This is, I guess, the main source of confusion that obscures some ontological or epistemological controversies. One should not confuse the invariance/objectivity/universality of the class with the asymmetry/subjectivity/conventional choice of one of its representation. As Debs and Redhead write

[...] the claim that these representations are inescapably based on conventional choices has been taken by many as a denial of their objectivity. As such, objectivity and conventionality in representation have often been framed as polar opposites.[...]

[However], a view of science that emphasizes the role of conventional choice need not be in conflict with a realist account of representation that allows for objectivity. One may maintain a cultural view of science and still be committed, as most realists are, to the existence of a single real ontology that humans inhabit (Debs and Redhead, 2007, Introduction, pp. 3 and 4).

I do not know if Born, when writing the above text, had also in mind Plato's famous allegory of the cave; however Weyl, in pursuing his philosophical reflections, clearly endeavours to reconcile realism with idealism (p. 117). Then, in almost exactly the same terms we discussed in $\S 3.5$, the distinction between the vector $v$ ("analogue of the objects in the real world") and its components $v^{\mu}$ ("analogues of the subjective phenomena") obtained by projection on a specific coordinate basis ("analogues of real observers"), Weyl concludes

Hence the model is the world of my phenomena and the absolute basis is that distinguished observer 'I' who claims that all phenomena are as they appear to him: on this level, object, observer and appearance all belong to the same world of phenomena, linked however by relations among which we can distinguish the 'objective' or invariant ones. Real observer and real object, I, thou, and the external world arise, so to speak, in unison and correlation with one another by subjecting the sphere of 'algebraic appearances' to the viewpoint of invariance. [...] The analogy renders the fact readily intelligible that the unique 'I' of pure consciousness, the source of meaning, appears under the viewpoint of objectivity as but a single subject among many of its kind (Weyl, 1949, § 17, pp. 123-124).

\subsection{Three open problems}

As far as contemporary physics is concerned, there exists of course an enormous literature on the subject of symmetry breaking. In addition, part III of Brading and Castellani's 2003 compilation explores interesting epistemological and philosophical implications of this concept. I will therefore not say more about these matters. Before we reach the conclusion, I would like to end this section by listing three open problems, that I consider to be crucial and more scientific than metaphysical (I believe that any kind of dualist approach cannot provide a satisfactory solution but just shifts the emphasis of the problems). All three seem pertained to the fourth facet of symmetry.

$\left(\mathrm{P}_{\mathrm{Q}}\right)$ The problem of quantum measurement: How does the interaction between a system $\mathcal{S}$ and a measurer $\mathcal{M}$ selects just one observed property or one observed event among the class of observable ones that a quantum state encompasses ? A satisfactory answer must come with a consistent and unified description of both $\mathcal{S}, \mathcal{M}$ and possibly $\mathcal{E}$ unlike the orthodox contemporary quantum theory which still attributes classical properties to $\mathcal{M}$.

$\left(\mathrm{P}_{\mathrm{N}}\right)$ The problem of now: In physics, all the points of the world line of a system are treated on an equal footing. In the space-time zone where intelligence has emerged, what does select a special thin slice of time we call present?

$\left(\mathrm{P}_{\mathrm{S}}\right)$ The problem of self: Among all the conscious minds, what does favour the special one you call your 
self?

As Born recalls about a quantum measurement

Expressed in mathematical terms the word projection is perfectly justified since the main operation is a direct generalisation of the geometrical act of projecting, only in a space of many, often infinitely many, dimensions (Born, 1953).

Some connections between these problems have been proposed. If Darwinian selection has not shaped our brains like a digital device, it has neither prepared us for a natural apprehension of the world at small scales. After one century of controversy on the quantum measurement issues, despite the recent progress on the understanding of the role played by environment (see note 8), we still do not know if we will be able to obtain, within a consistent theory, the reduction of a (possibly diagonalised by decoherence) density operator to a pure state or if this problem is irreducible any further because of our intrinsic limitations. This last line of pursuit follows a tradition initiated by Bohr and later endorsed by Wigner (1995, part III) and now defended by Penrose (1997), who proposed to relate $\mathrm{P}_{\mathrm{Q}}$ to $\mathrm{P}_{\mathrm{S}}$. It is worth noting that another approach of $\mathrm{P}_{\mathrm{S}}$ was proposed by Schrödinger (1944, Epilogue).

By the way, if we consider $\mathrm{P}_{\mathrm{S}}$ the opposite way by reversing its terms, viz. if we ask how we can infer the existence of other selves, we just face the old problem of other minds (Avramides, 2001). Nevertheless, I find the problem of self much more interesting than the problem of other minds because the latter finds naturally its answer in the necessity of escaping from a sterile kind of noological solipsism.

Even though quantum theory of fields offers the most brilliant and convenient way to reconcile special relativity and quantum theory, it leaves unchanged the problem of measurement. There seems to be a deep incompatibility between objective probabilities and a four-dimensional space-time. According to Everett 1957's multiworld interpretation, a bifurcation between universe branches occurs at each measurement. Had this attempt come with a proposal that clarifies the special status of measurement against pure quantum events, it would have ruled $\mathrm{P}_{\mathrm{Q}}$ out but at the cost of reinforcing the importance of $\mathrm{P}_{\mathrm{N}}$ and especially $\mathrm{P}_{\mathrm{S}}{ }^{22}$.

Many physical and philosophical discussions have been devoted to the arrow of time, but relatively few concern attempts to physically define the present (Hartle, 2005). Maybe questions $\mathrm{P}_{\mathrm{N}}$ and $\mathrm{P}_{\mathrm{S}}$ are two aspects of the same question, the first being formulated in physical terms while the other in neuropsychological terms.

A signature of a final theory should precisely be free of parameters with contingent value. Those who believe in the existence of such a theory should therefore add a fourth problem, possibly connected to the others with the help of anthropic selectionist arguments (see for instance Bostrom, 2010 and references therein), namely,

$\left(\mathrm{P}_{\mathrm{C}}\right)$ The problem of constants : How are the dimensionless constants like the coupling constants at low energy scales, some cosmological characteristics or even the dimension of space-time selected?

However, if we deny such an ultimate goal of getting rid of contingency, the importance of $\mathrm{P}_{\mathrm{C}}$ is weakened because it is the usual statement of how to digest an effective theory into the stomach of a wider one.

\section{Conclusion}

Throughout the twentieth century, the notion of symmetry has acquired extraordinary scope and depth in mathematics and, especially, in physics. In this paper I have proposed to recognise in this wide domain four intricately bound clusters each of them being scrutinised into some fine structure. First, this decomposition allowed us to examine closely the multiple different roles symmetry plays in many places in physics.

\footnotetext{
${ }^{22}$ It is noteworthy that to try to solve another old problem of choice, namely the problem of free will, Boussinesq has proposed in 1877 to connect it with the existence of bifurcations of the solutions of dynamical equations, attributing the choice of the branch not to an extreme sensitivity to "a very small change in the initial conditions" (Poincaré, 1959, chap. IV p. 76, see also p. 68) but to an external "guiding principle" (principe directeur) that establishes a fundamental clear-cut between inanimate and animate beings. If, on the contrary, we want to remain self-consistent within a unique materialistic continuum, the "guiding principles" that break the symmetry must definitely remain internal.
} 
Second, I have tried to unveil some relations with other disciplines like neurobiology, epistemology, cognitive sciences and, not least, philosophy. These excursions and the connections they reveal, that should gain to be investigated more thoroughly, offer, I hope an intellectually rewarding transversal journey.

To put in a shell many themes we encountered, I could not find a better shell than Valéry's; I cannot resist the temptation of offering it as the last words:

Like a pure sound or a melodic system of pure sounds in the midst of noises, so a crystal, a flower, a sea shell stand out from the common disorder of perceptible things. For us they are privileged objects, more intelligible to the view, although more mysterious upon reflection, than all those which we see indiscriminately. They present us with a strange union of ideas: order and fantasy, invention and necessity, law and exception. In their appearance we find a kind of intention and action that seem to have fashioned them rather as man might have done, but as the same time we find evidence of methods forbidden and inaccessible to us. We can imitate these singular forms; our hands can cut a prism, fashion an imitation flower, turn or model a shell; we are even able to express their characteristics of symmetry in a formula, or represent them quite accurately in a geometric construction. Up to this point we can share with "nature": we can endow her with designs, a sort of mathematics, a certain taste and imagination that are not infinitely different from ours; but then, after we have endowed her with all the human qualities she needs to make herself understood by human beings, she displays all the inhuman qualities needed to disconcert us... Valéry (1964).

Acknowledgements: It is a pleasure to thanks X. Bekaert, J. Le Deunff, E. Lesigne, K. Morand, K. Noui and L. Villain for precious discussions that allowed to selectively improve the formulation of some ideas presented here. I am also very grateful towards N. Mohammedi for his careful corrections.

\section{References}

Aristotle, 1938. Categories. On interpretation. Prior analytics. Loeb classical library, Harvard university press, Cambridge. Bilingual edition commented an translated from the Greek by Harold P. Cooke and Hugh Tredennick.

Arnold, V.I., 1984. Catastrophe Theory. SpringerVerlag, New York.

Auger, P., 1952. L'homme microscopique. Flammarion, Paris. 1966 for the 2nd edition.

Auletta, G., 2006. The controversial relationships between science and philosophy: a critical assessment. Libreria Editrice Vaticana, Vatican City.

Avramides, A., 2001. Other minds. The problems of philosophy, Routledge, New York.

Baars, B.J., Gage, N.M., 2010. Cognition, brain, and consciousness. Introduction to cognitive neuroscience. Academic Press, Burlington. 2nd edition.

Barchielli, A., Gregoratti, M., 2009. Quantum Trajectories and Measurements in Continuous Time. The diffusive Case. volume 782 of Lecture Notes in Physics. Springer, Dordrecht.

Batterman, R.W., 2010. Emergence, singularities, and symmetry breaking. Found. Phys. (On line first), $1-20$.

Bedau, M.A., Humphreys, P., 2008. Emergence: Contemporary readings in philosophy and science. a Bradford book, The MIT Press, Cambridge.

Belot, G., 2003. Notes on symmetries, in: Brading and Castellani (2003). pp. 393-412. pp. 393-412. Chap. 24.

Binétruy, P., 2006. Supersymmetry. Theory, experiment, and cosmology. Oxford University Press, Oxford.

Birkhoff, G.D., 1933. Aesthetic measure. Harvard University Press, Cambridge. See also Birkhoff (1956). 
Birkhoff, G.D., 1956. Mathematics of aesthetics, in: Newman, J.R. (Ed.), The world of mathematics, Dover Publications, Inc., New York. pp. 21852195. Vol. 4, part XXI.

Bokulich, A., Bokulich, P. (Eds.), 2010. Scientific structuralism. volume 281, Boston Studies in the Philosophy of Science. Springer, Dordrecht.

Borges, J.L., 1942. Ficciones. Grove Press, New York. Translated from the original Spanish by Anthony Bonner and edited by Anthony Kerrigan (1962).

Born, M., 1953. Physical reality. Philos. Quart. 3, 139-149. Reproduced as chap. 9 of Castellani (1998).

Bostrom, N., 2010. Anthropic bias. Observation selection effects in science and philosophy. Studies in philosophy, Routledge, New York.

Boswell, J., 1791/2008. The Life of Samuel Johnson. Penguin Classics, Penguin Books, London. Edited by David Womersley.

Bourbaki, N., 1968. Theory of sets. Elements of mathematics, Hermann, Paris. Translated from the French original edition Théorie des ensembles.

Boussinesq, J., 1877. Sur la conciliation de la liberté morale avec le déterminisme scientifique. C. R. Acad. Sci. 84, 362-364. Note by Adhémar-Jean-Claude Barré de Saint-Venant on the work reedited in Boussinesq (1922). Available on http://gallica.bnf.fr.

Boussinesq, J., 1922. Cours de physique mathématiques de la faculté des sciences. Complément au tome III, Conciliation du véritable déterminisme mécanique avec l'existence de la vie et de la liberté morale. Gauthier-Villars, Paris. Available on http://gallica.bnf.fr.

Brading, K., Brown, H.R., 2004. Are gauge symmetry transformations observable? British J. Philos. Sci. $55,645-665$.

Brading, K., Castellani, E., 2003. Symmetries in physics. Philosophical reflections. Cambridge University Press.
Breuer, H.P., Petruccione, F., 2002. The theory of open quantum systems. Oxford University Press, Oxford.

Bricmont, J., Sokal, A.P., 2001. Defense of a modest scientific realism, in: Knowledge and the world. Challenges beyond the science wars, Springer, Berlin. pp. 17-45.

Brink, D.M., 1965. Nuclear forces. volume 354 of The Commonwealth and international library. Selected readings in physics. Pergamon Press, Oxford.

Brown, J.H., West, G. (Eds.), 2000. Scaling in Biology. Proceedings volume in the Santa Fe Institute studies in the sciences of complexity Santa Fe Institute Studies in the Sciences of Complexity, Oxford University Press, New York.

Bruno, G., 1584/1995. La cena de le ceneri — The ash wednesday supper. volume 4 of Renaissance Society of America. University of Toronto Press, Toronto. Translated from the original Italian by Edward A. Gosselin and Lawrence S. Lerner.

Butterfield, J., 2005. On the persistence of particles. Found. Phys. 35, 233-269.

Butterfield, J., 2010. Less is different: Emergence and reduction reconciled. Found. Phys. (On line first), 1-71.

Butterworth, B., 1999. The mathematical brain. Macmillan, London. US edition (Free Press) is entitled: What counts. How every brain is hardwired for math.

Campbell, D.T., 1959. Methodological suggestions from a comparative psychology of knowledge processes. Inquiry 2, 152-182.

Campbell, D.T., 1960. Blind variation and selective retention in creative thought as in other knowledge processes. Psychological Review 67, 380-400. Reprinted as chap. III of Radnitzky and Bartley (1987).

Campbell, D.T., 1974. Evolutionary epistemology, in: Schilpp, P.A. (Ed.), The philosophy of Karl 
Popper, Open Court, La Salle, Illinois. pp. 413463. Reprinted as chap. II of Radnitzky and Bartley (1987). For an updated account of Campbell's influence see Heyes and Hull (2001).

Carnap, R., 1966. Philosophical foundations of physics. An introduction to the philosophy of science. Basic Books Inc., New York.

Cassirer, E., 1938. Le concept de groupe et la théorie de la perception. Journal de Psychologie normale et pathologique $35,368-414$.

Cassirer, E., 1944. The concept of group and the theory of perception. Philosophy and phenomenological research V, 1-36. Translated by Aron Gurwitsch from the original French Cassirer (1938).

Castellani, E. (Ed.), 1998. Interpreting bodies. Classical and quantum objects in modern physics, Princeton University Press, Princeton.

Castellani, E., 2002. Reductionism, emergence and effective field theories. Stud. Hist. Philos. Mod. Phys. 33, 251-267.

Castellani, E., 2003. Symmetry and equivalence, in: Brading and Castellani (2003). pp. 425-436. pp. 425-436. Chap 26.

Chandrasekhar, S., 1987. Truth and beauty. Aesthetics and motivations in science. The University of Chicago Press, Chicago.

Changeux, J.P., 1985. Neuronal man. The biology of mind. Princeton University Press, Princeton. Translation by Laurence Garey from the French original edition L'homme neuronal (1983, Fayard).

Changeux, J.P., 2002. The physiology of truth. Neuroscience and human knowledge (mind/brain/behaviour initiative). Belknap Press of Harvard University Press, Cambridge. Translation by M. B. DeBevoise from the French original edition L'homme de vérité (Odile Jacob).

Changeux, J.P., 2012. The Good, the True, and the Beautiful: A Neuronal Approach. Yale University Press, New haven and London. Translated and revised by Laurence Garey from the French original edition Du vrai, du beau, du bien : une nouvelle approche neuronale (2008, Odile Jacob).

Changeux, J.P., Courrège, P., Danchin, A., 1973. A theory of the epigenesis of neuronal networks by selective stabilization of synapses. Proc. Nat. Acad. Sci. U.S.A. 70, 2974-2978.

Choquet-Bruhat, Y., DeWitt-Morette, C., 1982. Analysis, manifolds and physics. Elsevier science, Amsterdam. Revised Edition (Part I).

Clark, A., 2008. Supersizing the mind. Embodiment, action, and cognitive extension. Oxford University Press, Oxford.

Cornwell, J.F., 1984. Group theory in physics. Academic Press, London. 2 vols.

Cournot, A.A., 1851/1956. An Essay on the foundations of our knowledge. The Liberal Arts Press, New York. Translated by Merritt $H$. Moore from the original French Essai sur les fondements de nos connaissances et sur les caractères de la critique philosophique available on http://gallica.bnf.fr.

Cournot, A.A., 1861. Traité de l'enchaînement des idées fondamentales dans les sciences et dans l'histoire. Hachette, Paris. Available on http://gallica.bnf.fr.

Curie, P., 1894. Sur la symétrie dans les phénomènes physiques, symétrie d'un champ électrique et d'un champ magnétique. J. de Physique (3ème série) III, 393-415. Available on http://gallica.bnf.fr. English translation in (Rosen, 1982, pp. 17-25).

Curtin, D.W., 1980. The aesthetic dimension of science. Nobel Conference, Philosophical library, New York.

Cusa, N., 1440/1985. On learned ignorance. A translation and an appraisal of De Docta Ignorantia. Arthur J. Banning Press, Minneapolis. Translated from the original Latin by Germain Heron.

Dawkins, R., 1976. The selfish gene. Oxford University Press, Oxford. 
Debs, T.A., Redhead, M., 2007. Objectivity, invariance, and convention. Symmetry in physical science. Harvard University Press, Cambridge.

Dehaene, S., 1997. The number sense. How the mind creates mathematics. Oxford University Press, Oxford.

Delahaye, J.P., 1999. Information, complexité et hasard. Hermès Science Publications, Paris.

Demazure, M., 2000. Bifurcations and catastrophes. Geometry of solutions to nonlinear problems. Universitext, Springer, Berlin. Translated by David Chillingworth from the French edition Catastrophes et bifurcations (Ellipses, 1989).

Dennett, D.C., 1996. Kinds of minds. Toward an understanding of consciousness. Science Masters Series, Basic Books, New York.

Dirac, P.A.M., 1926. On the theory of quantum mechanics. Proc. Roy. Soc. London Ser. A 112, 661677.

Dirac, P.A.M., 1930. A theory of electrons and protons. Proc. Roy. Soc. London Ser. A 126, 360-365.

Dirac, P.A.M., 1963. The evolution of the physicist's picture of nature. Sci. Amer. 208, 45-53.

Dirac, P.A.M., 1982. Pretty mathematics. Internat. J. Theoret. Phys. 21, 603-605.

Duck, I., Sudarshan, E.C.G., 1997. Pauli and the spin-statistics theorem. World Scientific, Singapore.

Duhem, P., 1906/1954. The aim and structure of physical theory. Princeton University Press, Princeton. Translated by Philip P. Wiener from the French original edition La théorie physique, son objet, sa structure (Chevalier et Rivière, 1906).

Edelman, G.M., 1978. Group selection and phasic reentrant signalling: a theory of higher brain function, in: Edelman, G.M., Mountcastle, V.B. (Eds.), Mindful brain. Cortical organization and the group-selective theory of higher brain function, The MIT Press, Cambridge. p. 51.
Edelman, G.M., 1987. Neural darwinism. The theory of neuronal group selection. Basic Books, New York.

Edelman, G.M., 1992. Bright air, brilliant fire. On the matter of the mind. BasicBooks, New York.

Edelman, G.M., 2006. Second nature. Brain science and human knowledge. Yale University Press, New Haven.

Einstein, A., 1936. Physics and reality. J. Franklin Inst. 221, 349-382. Translated by Jean Piccard from the original german text pp. 313-347.

Einstein, A., 1949. Reply to criticisms, in: Schilpp, P.A. (Ed.), Albert Einstein: Philosopher scientist, Open Court, La Salle, Illinois. pp. 665-688.

Esfeld, M., 2006. Scientific realism and the history of science, in: Auletta (2006). pp. 251-275. pp. 251275 .

Everett III, H., 1957. "Relative state" formulation of quantum mechanics. Rev. Modern Phys. 29, 454462. Reproduced as $\S$ II.3 of Wheeler and Zurek (1983).

Feigl, H., Maxwell, G. (Eds.), 1962. Scientific explanation, space, and time. volume III of Minnesota Studies in the Philosophy of Science, University of Minnesota press, Minneapolis. http://www.mcps . umn. edu/philosophy/complete.html.

Feigl, H., Scriven, M. (Eds.), 1956. Foundations of science and the concepts of psychology and psychoanalysis. volume I of Minnesota Studies in the Philosophy of Science, University of Minnesota press, Minneapolis. http://www.mcps . umn. edu/philosophy/complete.html.

Feyerabend, 1962. Explanation, reduction, and empiricism, in: Feigl and Maxwell (1962). pp. 28-97. pp. 28-97. http://www .mcps . umn.edu/philosophy/complete.html.

Feynman, R.P., Leighton, R.B., Sands, M., 1970. The Feynman lectures on physics. Addison Wesley publishing company, Reading, Massachusetts. 
Fine, A., 1996. The shaky game. Einstein realism and the quantum theory. The University of Chicago Press, Chicago. 2nd edition.

Fonda, L., Ghirardi, G.C., 1970. Symmetry principles in quantum physics. Theoretical Physics, vol. 1, Marcel Dekker, New York.

van Fraassen, B.C., 1989. Laws and symmetry. Oxford University Press, Oxford.

Fresnel, A., 1818. Mémoire sur la diffraction de la lumière. Mem. de l'Acad. sc. Inst. France 5, 339-475.

http://www.academie-sciences.fr/activite/archive/dossiers/Fresnel/Fresnel_publi.htm

Galilei, G., 1632. Dialogo sopra i due massimi sistemi del mondo. Le opere di Galileo Galilei, Edizione Nazionale, vol. VII (1897) under the direction of Antonio Favaro, G. Barbera, Firenze.

Galilei, G., 1632/1981. Dialogue concerning the two chief world systems: Ptolemaic and Copernican. The Modern Library, New York. Translated from the original Italian by Stillman Drake.

Gibbs, J.W., 1902/2010. Elementary principles in statistical mechanics: developed with especial reference to the rational foundation of thermodynamics. Cambridge University Press, Cambridge. Reprint of original 1902 edition.

Giedymin, J., 1982. Science and Convention. Essays on Henri Poincaré's Philosophy of Science and the Conventionalist Tradition. Foundations \& Philosophy of Science \& Technology, Pergamon Press, Oxford.

Giedymin, J., 1992. Conventionalism, the pluralist conception of theories and the nature of interpretation. Stud. Hist. Philos. Sci. 23, 423-443.

Giulini, D., Joos, E., Kiefer, C., Kupsch, J., Stamatescu, I.O., Zeh, H.D., 2003. Decoherence and the Appearance of a Classical World in Quantum Theory. Springer-Verlag, Berlin. (2nd edition).

Hacyan, S., 2009. Geometry as an object of experience: İActhe missed debate between Poincaré and Einstein. Eur. J. Phys. 30, 337-343.
Hadamard, J., 1954. Essay on the psychology of invention in the mathematical field. Dover Publications, New York. Cambridge University Press (1945) for the original first edition.

Haldane, J.B.S., 1927. Possible worlds and other essays. Chatto and Windus, London.

Hamilton, W., 1861. Discussions on philosophy and literature. Harper and Brothers, New York.

Hanson, N.R., 1958. Patterns of discovery. An inquiry into the conceptual foundations of science. Cambridge University Press, Cambridge.

Hardy, G.H., 1940. A mathematician's apology. Cambridge: University Press, Cambridge. http://www.math.ualberta.ca/mss/.

Hartle, J.B., 2005. The physics of 'Now'. Amer. J. Phys. 73, 101-109.

Heisenberg, W., 1926. The many-body problem and resonance in quantum mechanic. Zts. f. Phys. 38, 411-426. English translation in Duck and Sudarshan (1997) pp.115-125.

Heisenberg, W., 1927. The many-body problem and resonance in quantum mechanic II. Zts. f. Phys. 40, 239-267.

Heisenberg, W., 1932. On the structure of atomic nuclei, I. Zts. f. Phys. 77, 1-11. English translation in Brink (1965) pp. 144-154.

Heisenberg, W., 1958. Physics and philosophy. The revolution in modern science. volume 1958 of World perspectives. Harper and brothers, New York.

Heisenberg, W., 1974. Across the frontiers. volume 1974 of World perspectives. Harper and Rows, New York.

Henshilwood, C.S., d'Errico, F., Yates, R., Jacobs, Z., Tribolo, C., Duller, G.A.T., Mercier, N., Sealy, J.C., Valladas, H., Watts, I., Wintle, A.G., 2002. Emergence of modern human behavior: Middle stone age engravings from South Africa. Science $295,1278-1280$. 
Heyes, C.M., Hull, D.L. (Eds.), 2001. Selection theory and social construction. The evolutionary naturalistic epistemology of Donald T. Campbell. Suny Series in Philosophy and Biology, State University of New York Press, New York.

Hon, G., Goldstein, B.R., 2008. From summetria to symmetry: The making of a revolutionary scientific concept. volume 20 of Archimedes. Springer.

Houtappel, R.M.F., Van Dam, H., Wigner, E.P., 1965. The conceptual basis and use of the geometric invariance principles. Rev. Modern Phys. 37, 595-632.

Hull, D.L., 2001. Science and Selection: Essays on Biological Evolution and the Philosophy of Science. Cambridge Studies in Philosophy and Biology, Cambridge University Press, Cambridge.

Hume, D., 1758/1999. An enquiry concerning human understanding. Oxford Philosophical Texts, Oxford University Press, Oxford. Edited by Tom L. Beauchamp.

Ismael, J., van Fraassen, B.C., 2003. Symmetry as a guide to superfluous theoretical structure, in: Brading and Castellani (2003). pp. 371-392. pp. 371-392. Chap. 23.

James, W., 1880. Great men, great thoughts, and the environment. Atlantic Monthly 46, 441-459.

Jammer, M., 1994. Concepts of space. The history of theories of space in physics. Dover Publications, New York.

Jeffreys, H., 1931. Scientific inference. Cambridge University Press, Cambridge.

Jones, H.F., 1998. Groups, representations and physics. Institute Of Physics Publishing, Bristol.

Kadanoff, L.P., 2000. Statistical physics. Statics, dynamics and renormalization. World Scientific, Singapore.

Kant, I., 1781/1998. Critique of pure reason. Cambridge University Press, Cambridge. Edited and translated by Paul Guyer and Allen W. Wood.
Klein, F., 1893. A comparative review of recent researches in geometry. Bull. New York Math. Soc. 2, 215-249. Translated by M. W. Haskell from the German original (1872).

Kosmann-Schwarzbach, Y., 2010. The Noether theorems. Invariance and conservation laws in the twentieth century (sources and studies in the history of mathematics and physical sciences). Springer. Translated by Bertram E. Schwarzbach from the French original edition (Les Éditions de l'École Polytechnique, 2004).

Kosso, P., 2003. Symmetry, objectivity and design, in: Brading and Castellani (2003). pp. 413-424. pp. 413-424. Chap 25.

Kuhn, T., 1970. The structure of scientific revolutions. The University of Chicago Press, Chicago. 2nd edition.

Laertius, D., 1925. Lives of eminent philosophers (vol. 2, books 6-10). Loeb classical library, Harvard university press, Cambridge. Bilingual edition commented and translated from the Greek by R. D. Hicks.

Landauer, R., 1967. Wanted: a physically possible theory of physics. IEEE Spectrum 4, 105-109.

Landauer, R., 1999. Information is a physical entity. Physica A 263, 63-67.

Langevin, P., 1923. La physique depuis vingt ans. Gaston Douin, Paris.

Laudan, L., 1981. A confutation of convergent realism. Philos. Sci. 48, 19-49. Reprinted as chap. VI of Papineau (1996).

Laudan, L., 1984. Realism without the real. Philos. Sci. 51, 156-162.

Laudan, L., 1990. Demystifying underdetermination, in: Savage (1990). pp. 267-297. pp. 267-297. http://www.mcps . umn.edu/philosophy/complete.html. reproduced as chap. 2 of Laudan (1996). 
Laudan, L., 1996. Beyond positivism and relativism. Theory, method, and evidence. Westview Press, Boulder.

Leff, H.S., Rex, A.F. (Eds.), 2003. Maxwell's demon 2. Entropy, classical and quantum information, computing, Institute of Physics Publishing, Bristol.

Lévi-Strauss, C., 1968. The savage mind. The University of Chicago Press, Chicago. Translated from the original French La pensée sauvage (Plon, 1962).

Lewis, P.J., 2001. Why the pessimistic induction is a fallacy. Synthese 129, 371-380.

Li, M., Vitányi, P., 2008. An introduction to Kolmogorov complexity and its applications. Texts in Computer Science, Springer, New York. 3rd edition.

Lipscomb, W.N., 1980. Aesthetic aspect of science, in: Curtin (1980). pp. 1-24. pp. 1-24.

Llinás, R.R., 2001. I of the vortex. From neurons to self. The MIT Press, Cambridge.

Mach, E., 1906/1976. Knowledge and Error. Sketches on the Psychology of Enquiry. volume 3 of $\mathrm{Vi}$ enna Circle Collection. Reidel Publishing Compagny, Dordrecht. Translated from the 5th edition of Erkenntnis und Irrtum from the German by T. J. McCormack and P. Foulkes.

Machery, É., 2009. Doing without concepts. Oxford University Press, New York.

Margolis, E., Laurence, S., 2007. The ontology of concepts-abstract objects or mental representations? NOÛS 41, 561-593.

Maxwell, G., 1962. The ontological status of theoretical entities, in: Feigl and Maxwell (1962). pp. 3-27. pp. 3-27. http://www.mcps . umn.edu/philosophy/complete.html.

McAllister, J.W., 1996. Beauty and revolution in science. Cornell University Press, Ithaca.
Moriyasu, K., 1983. An elementary primer for gauge theory. World Scientific, Singapore.

Morrison, P., 1995. Nothing is too wonderful to be true. volume 11 of Masters of Modern Physics. American Institute of Physics Press, New York.

Mouchet, A., 2013a. An alternative proof of Wigner theorem on quantum transformations based on elementary complex analysis. Phys. Lett. A 377, 2709-2711.

Mouchet, A., 2013b. L'élégante efficacité des symétries. UniverSciences, Dunod, Paris.

Mouchet, A., 2013c. . Eur. Phys. J. H 38, 661-702.

Murphy, G.L., 2002. The big book of concepts. The MIT Press, Cambridge.

von Neumann, J., 1932/1955. Mathematical foundations of quantum mechanics. Princeton University Press, Princeton. Translated by Robert T. Beyer from the original German edition (1932).

Nozick, R., 2001. Invariances. The structure of the objective world. The Belknap Press of Harvard university press, Cambridge.

Papineau, D., 1996. The philosophy of science. Oxford Readings in Philosophy, Oxford University Press, Oxford.

Penrose, R., 1974. The rôle of aesthetics in pure and applied mathematical research. Bull. Inst. Math. Appl. 10, 266-271.

Penrose, R., 1980. On schwarzschild causality-a problem for "lorentz covariant" general relativity, in: Tipler, F.J. (Ed.), Essays in general relativity: a festschrift for Abraham Taub, Academic Press, New York. pp. 1-12.

Penrose, R., 1997. The large, the small and the human mind. Cambridge University Press, Cambridge.

Platek, S.M., Keenan, J.P., Shackelford, T.K., 2007. Evolutionary cognitive neuroscience. The MIT Press, Cambridge. 
Poincaré, H., 1891. Les géométries non euclidiennes. Rev. Gén. Sci. Pur. Appl 2, 769-774. Reproduced with modifications in chap. III of Poincaré (1952) and the section Geometry and astronomy is reproduced in chap. $\mathrm{V}$.

Poincaré, H., 1895. L'espace et la géométrie. Revue de métaphysique et de morale 3, 631-646. Reproduced with modifications in chap. IV of Poincaré (1952).

Poincaré, H., 1900a. Les relations entre la physique expérimentale et la physique mathématique. Rev. Gén. Sci. Pur. Appl 11, 1163-1175. English translation in Poincaré (1900b).

Poincaré, H., 1900b. The relations between experimental and mathematical physics. Sci. Amer. 83, 282. Reproduced in chapters IX et X of Poincaré (1952).

Poincaré, H., 1902. Sur la valeur objective de la science. Revue de métaphysique et de morale 10, 263 293. Reproduced as chap. X and XI of Poincaré (1958).

Poincaré, H., 1903. L'espace et ses trois dimensions. Revue de métaphysique et de morale 11, 281-301. Reproduced as chap. III of Poincaré (1958).

Poincaré, H., 1908. L'invention mathématique. Bulletin de l'institut général psychologique 8ième année, mai-juin (3), 4-15. Reproduced as chap. III of Poincaré (1959).

Poincaré, H., 1911. L'évolution des lois. Scientia (Rivista di Scienza) 9, 275-292. Reproduced as chap. I of Poincaré (1913) without the section based on differential equations.

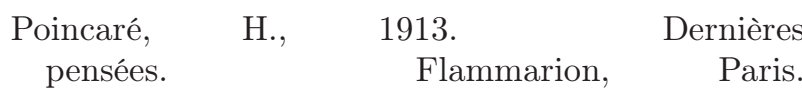
www.ac-nancy-metz.fr/enseign/philo/textesph/\#poinca.

Poincaré, H., 1952. Science and hypothesis. Dover Publications, Inc., New York. Translated by W. J. Greenstreet from the French La science et l'hypothèse (Flammarion, 1902).
Poincaré, H., 1958. The Value of Science. Dover Publications, Inc., New York. Translated by George Bruce Halsted from the French La valeur de la science (Flammarion, 1905).

Poincaré, H., 1959. Science and method. Dover Publications, Inc., New York. Translated by Francis Maitland from the French Science et méthode (Flammarion, 1908).

Popper, K., 1959. The logic of scientific discovery. Routledge, London.

Popper, K., 1969. Conjectures and refutations. The growth of scientific knowledge. Routledge, London. 3rd edition.

Popper, K., 1978. Natural selection and the emergence of mind. Dialectica 32, 339-355.

Popper, K., 1979. Objective knowledge. An evolutionary approach. Oxford University Press, Oxford. Revised edition of the original edition (1972).

Poston, T., Stewart, I., 1978. Catastrophe theory and its applications. Pitman, London.

Presilla, C., Onofrio, R., Tambini, U., 1996. Measurement quantum mechanics and experiments on quantum zeno effect. Ann. Physics 248, 95-121.

Psillos, S., 1999. Scientific realism. How science tracks truth. Routledge, London.

Putnam, H., 1975. Mathematics, matter and method. volume 1 of Philosophical Papers. Cambridge University Press, Cambridge. 2nd ed.

Putnam, H., 1978. Meaning and the moral sciences. Routledge and Kegan Paul, London.

Radnitzky, G., Bartley, W.W., 1987. Evolutionary epistemology, rationality, and the sociology of knowledge. Open Court Publishing Company, Peru.

Reichenbach, H., 1938. Experience and prediction. An analysis of the foundations and the structure of knowledge. The University of Chicago Press, Chicago. 
Rosch, E., 1978. Principles of categorization, in: Rosch, E., Lloyd, B.L. (Eds.), Cognition and Categorization, Lawrence Erlbaum Associates, Hillsdale. pp. 27-48.

Rosen, J., 1982. Symmetry in physics. Selected reprints. American Association of Physics Teachers, Melville.

Russell, B., 1917. Mysticism and logic, and other essays. George Allen and Unwin, London. Original publication in New Quaterly (November 1907).

Russell, B., 1919. Introduction to mathematical philosophy. George Allen and Unwin, London. Republished by Dover (1993).

Sankey, H., 2001. Scientific realism: An elaboration and a defence. Theoria 98, 35-54. Reproduced in chapter 1 of Sankey (2008).

Sankey, H., 2008. Scientific Realism and the Rationality of Science. Ashgate, Burlington.

Savage, C.W. (Ed.), 1990. Scientific theories. volume XIV of Minnesota Studies in the Philosophy of Science, University of Minnesota press, Minneapolis. http://www.mcps . umn.edu/philosophy/complete.html.

Schlosshauer, M., 2007. Decoherence and the quantum-to-classical transition. The frontiers collection, Springer, Berlin.

Schrödinger, E., 1944. What is life. Cambridge University Press, Cambridge.

Segerstråle, U., 2000. Defenders of the Truth. The battle for science in the sociobiology debate and beyond. Oxford University Press, Oxford.

Sellars, W., 1956. Empiricism and the philosophy of mind, in: Feigl and Scriven (1956). pp. 253-329. pp. 253-329. http://www.mcps . umn.edu/philosophy/complete.html. reprinted as chap. 5 of Sellars (1991).

Sellars, W., 1991. Science, perception and reality. Ridgeview Publishing Compagny, Atascadero.
Seth, A.K., Baars, B.J., 2005. Neural Darwinism and consciousness. Consciousness and Cognition 14, 140-168.

Shanahan, T., 2004. The evolution of Darwinism. Selection, adaptation, and progress in evolutionary biology. Cambridge University Press, Cambridge.

Simon, R., Mukunda, N., Chaturvedi, S., Srinivasan, V., 2008. Two elementary proofs of the Wigner theorem on symmetry in quantum mechanics. Phys. Lett. A 372, 6847-6852.

de Smit, B., Lenstra, H.W., 2003. The mathematical structure of escher's print gallery. Notices Amer. Math. Soc. 50, 446-451.

Smith, E.E., 1998. Concepts and categorization, in: Smith and Osherson (1995). pp. 3-33. pp. 3-33. Chap. 1.

Smith, E.E., Osherson, D.N. (Eds.), 1995. Thinking. An Invitation to Cognitive Science (2nd ed.), vol. 3:, The MIT Press, Cambridge.

Sokal, A.P., Bricmont, J., 1998. Fashionable nonsense. Postmodern intellectuals'abuse of science. Picador, New York.

Sternberg, S., 1994. Group theory and physics. Cambridge University Press, Cambridge.

Stump, D., 1991. Poincaré's thesis of the translatability of euclidean and non-euclidean geometries. NOÛS 25, 639-657.

Taine, H., 1872. On intelligence. Holt and Williams, New York. Translated by T. D. Haye from the French De l'intelligence (1870).

Tarasov, L., 1986. This Amazingly Symmetrical World. Symmetry around us, symmetry at the heart of everything. Mir Publishers, Moscow. Translated from Russian edition (1982) by Alexander Repyev.

Toulmin, S.E., 1967. The evolutionary development of natural science. Amer. Sci. 55, 456-471. 
Toulmin, S.E., 1972. Human understanding. The collective use and evolution of concepts. Princeton University Press, Princeton.

Ullmo, J., 1969. La pensée scientifique moderne. Flammarion, Paris.

Utiyama, R., 1956. Invariant theoretical interpretation of interaction. Phys. Rev. 101, 1597-1607.

Valéry, P., 1964. Aesthetics. Bollinger Series XLV, vol. 13, Pantheon Books, New York. Man and the Sea Shell translated by Ralph Manheim from the French L'homme et la coquille (Variété V, Gallimard).

Weil, A., 1960. De la métaphysique aux mathématiques. Sciences , 52-56Reproduced pp. 408-412 in Weil (1979).

Weil, A., 1979. Euvres scientifiques. Collected papers, vol. II: 1951-1964. Springer-Verlag, New York.

Weinberg, S., 1992. Dreams of a final theory. The scientist's search for the ultimate laws of nature. Pantheon Books, New York.

Weinberg, S., 1995. The quantum theory of fields (vol. I : Foundations). Cambridge University Press, New York.

Weinberg, S., 2000. The quantum theory of fields (vol. III : Supersymmetry). Cambridge University Press, New York.

Weyl, H., 1949. Philosophy of mathematics and natural sciences. Princeton University Press, Princeton. Revised and augmented English edition translated by Olaf Helmer from the German.

Weyl, H., 1952. Symmetry. Princeton University Press, Princeton. Traduction française : Symétrie et mathématique moderne, 1964, Flammarion, Paris.

Wheeler, J.A., Zurek, W.H. (Eds.), 1983. Quantum theory and measurement, Princeton series in physics. Princeton University Press, Princeton.
Wigner, E.P., 1960. The unreasonable effectiveness of mathematics in the natural sciences. Comm. Pure Appl. Math. 13, 1-14.

Wigner, E.P., 1995. Philosophical reflections and syntheses. Springer, Berlin. Annoted by Gérard G. Emch.

Worrall, J., 1989. Structural realism: The best of both worlds? Dialectica 43, 99-124. Reprinted as chap. VII of Papineau (1996).

Yang, C.N., 1980. Beauty and theoretical physics, in: Curtin (1980). pp. 25-40. pp. 25-40.

Yang, C.N., 1994. Conceptual beginnings of various symmetries in twentieth century physics. Chinese J. Phys. 32, 1437-1446.

Yang, C.N., Mills, R.L., 1954. Conservation of isotopic spin and isotopic gauge invariance. Phys. Rev. 96, 191-195.

Yourcenar, M., 1976. The abyss. Farrar, Straus and Giroux, New York. English translation by Grace Frick in collaboration with the author from the French original edition L'œuvre au noir (Gallimard, 1968).

Zellner, A., Keuzenkamp, H.A., McAleer, M. (Eds.), 2001. Simplicity, inference and modelling. Keeping it sophisticatedly simple. Cambridge University Press, Cambridge. 